Research Article

\title{
Image Denoising of Adaptive Fractional Operator Based on Atangana-Baleanu Derivatives
}

\author{
Xiaoran Lin, ${ }^{1}$ Yachao Wang $\mathbb{D}^{1},{ }^{1}$ Guohao $\mathrm{Wu}^{2}$ and Jing Hao ${ }^{1}$ \\ ${ }^{1}$ College of Information Technology, Hebei University of Economics and Business, Shijiazhuang, Hebei 050061, China \\ ${ }^{2}$ College of Computer Science, Chongqing University, Chongqing 40044, China
}

Correspondence should be addressed to Yachao Wang; wangyachao@cqu.edu.cn

Received 15 January 2021; Revised 22 February 2021; Accepted 12 March 2021; Published 7 April 2021

Academic Editor: Zakia Hammouch

Copyright (c) 2021 Xiaoran Lin et al. This is an open access article distributed under the Creative Commons Attribution License, which permits unrestricted use, distribution, and reproduction in any medium, provided the original work is properly cited.

A fractional integral operator can preserve an image edge and texture details as a denoising filter. Recently, a newly defined fractional-order integral, Atangana-Baleanu derivatives (ABC), has been used successfully in image denoising. However, determining the appropriate order requires numerous experiments, and different image regions using the same order may cause too much smoothing or insufficient denoising. Thus, we propose an adaptive fractional integral operator based on the Atangana-Baleanu derivatives. Edge intensity, global entropy, local entropy, and local variance weights are used to construct an adaptive order function that can adapt to changes in different regions of an image. Then, we use the adaptive order function to improve the masks based on the Grumwald-Letnikov scheme (GL_ABC) and Toufik-Atangana scheme (TA_ABC), namely, Ada_GL_ABC and Ada_TA_ABC, respectively. Finally, multiple evaluation indicators are used to assess the proposed masks. The experimental results demonstrate that the proposed adaptive operator can better preserve texture details when denoising than other similar operators. Furthermore, the image processed by the Ada_TA_ABC operator has less noise and more detail, which means the proposed adaptive function has universality.

\section{Introduction}

The theory of fractional-order derivatives has been applied in many fields, such as physics, fluid mechanics, physiology, medical science, and epidemic diseases [1-4]. With the development of information science, fractional operators have gained incomparable advantages over integral operators in many fields. A fractional derivative recurrent neural network can effectively improve estimation accuracy in parameter identification [5]. Complex behaviors in fractional-order financial systems can provide theoretical basis for the government [6]. Fractional-order control systems perform more accurately and elegantly than traditional systems [7]. In signal processing, the characteristics of fractional differential operators, such as "nonlocality," "memorability," and "weak derivatives," are also applied [8-10]. These properties can improve the high frequency of an image while preserving the performance of the low and medium frequencies. In other words, methods based on fractional calculus for enhanced images can enhance the texture details while preserving the texture details of the smooth region in images [11, 12]. Therefore, many scholars are engaged in research on the application of fractional operators in image enhancement and denoising. The most representative scholar is Y.F. Pu, who, with his team, constructed image enhancement and denoising operators by fractional calculus $[10,13]$. Based on the Grunwald-Letnikov (GL) approximation, a medical image enhancement method was proposed by Guan et al. [14]. An adaptive image enhancement operator based on fractionaldifferential and image gradient feature was proposed by Lan [15]. Arian Azarang inferred different structure mask to image fusion [16]. An adaptive fractional-order integral filter was presented for echocardiographic image denoising [17].

The basic theory of abovementioned fractional operators is mainly the definitions of GL and Riemann-Liouville (RL). The Caputo derivative is another definition of fractional order that is widely studied and applied; it includes the numerical solutions of fractional equations and the 
properties of systems $[18,19]$. New fractional derivatives and applications based on the frame of the Caputo derivative have received much attention from experts. The existence and stability of Belouso-Zhabotinskii reaction systems with Atangana-Baleanu fractional-order derivatives are discussed in [20]. In [21], the locally and globally asymptotically stable of symbiosis system modelling by the Atangana-Baleanu derivative are analyzed. With the development of research, fractional-differential operators with nonlocal and nonsingular kernels are used to image filters [22-24]. Furthermore, an AB-fractional differential mask based on the Gaussian kernel has been introduced to detect blood vessels in retinal images [25]. Behzad Ghanbari and Abdon Atangana designed an ABC-fractional derivatives mask that is used for image denoising. The $\mathrm{ABC}$-fractional derivatives mask is computationally efficient and has excellent performance in the denoising of nosy images [26]. In the process of denoising, many experiments are required to determine the order of the mask. Moreover, because using a fixed order may lead to excess or deficiency for denoising effect, an adaptive fractional operator based on Atangana-Baleanu derivatives is proposed in this paper, which is called Ada_GL_ABC. We consider the gradient of the image, local entropy, global entropy, and local variance weights to construct a function for solving the adaptive order. The starting point of this idea is removing the image noise while preserving the edge and texture details of the image as much as possible. The adaptive function proposed by us is different from that of other studies. We consider both global and local information, the adaptive function contains more comprehensive information when determining the order, and the order used for denoising is more appropriate. And, the adaptive function designed by us has a certain generality. The function can be applied not only to GL_ABC mask but also to TA_ABC mask, which is rarely seen in the previous literature.

The remainder of this paper is organized as follows: the basic definitions of fractional derivatives and the structure of fractional-masks are introduced in Section 2. In Section 3, the function of the adaptive fractional-order integral operator based on Atangana-Baleanu derivatives is described. The performance of the proposed adaptive operator is discussed in Section 4. In Section 5, the conclusions are elaborated.

\section{Preliminaries}

2.1. Definitions of the Fractional Derivatives. Many basic definitions of fractional derivatives exist [27]. Recently, the
Mittag-Leffler function was introduced to compute fractional derivatives. This new definition is named the Atangana-Baleanu fractional derivative; it is based on the definition of Liouville-Caputo (ABC) and can be defined as follows [28]:

$$
{ }_{0}^{A B C} D_{t}^{\gamma} f(t)=\frac{A(\gamma)}{1-\gamma} \int_{0}^{t} E_{\gamma}\left[-\beta \frac{(t-\tau)^{\gamma}}{1-\gamma}\right] f \dot{(\tau)} \mathrm{d} \tau, \quad 0<\gamma \leq 1 .
$$

The Atangana-Baleanu fractional integral with order $\beta$ can be depicted as

$$
\begin{aligned}
{ }_{0}^{A B C} J_{t}^{\gamma} f(t)= & \frac{1-\gamma}{A(\gamma)} f(t) \\
& +\frac{\gamma}{\Gamma(\gamma) A(\gamma)} \int_{0}^{T} f(\tau)(t-\tau)^{\gamma-1} \mathrm{~d} \tau, \quad 0<\gamma \leq 1,
\end{aligned}
$$

where $A(*)$ is a normalization function, and this function satisfies $A(0)=A(1)=1$. It can be described by

$$
A(\gamma)=1-\gamma+\frac{\gamma}{\Gamma(\gamma)}
$$

The $\mathrm{ABC}$ derivative inherits the memory of the Mittag-Leffler function, which, with index $\gamma$, is denoted as

$$
E_{\gamma}(t)=\sum_{k=0}^{\infty} \frac{t^{k}}{\Gamma(\gamma k+1)}, \quad \gamma>0 .
$$

The GL definition is one of the best-known definitions of discrete fractional calculus and is widely applied to image processing. Details of the GL definition are expounded in Definition 1.

Definition 1. The GL definition of fractional calculus formula with $\alpha$-order of [29] is described as

$$
{ }_{0} D_{b}^{\alpha} f(x)=\lim _{h \rightarrow 0} \frac{1}{h^{\alpha}} \sum_{j=0}^{[(b-a) / h]}(-1)^{j}\left(\begin{array}{l}
\beta \\
j
\end{array}\right) f(x-j h),
$$

where $h$ is the step, [*] represents the rounded operation, $\left(\begin{array}{c}\beta \\ j\end{array}\right)=((\Gamma(\beta+1)) /(j ! \Gamma(\beta-j+1)))$, and $\Gamma(\beta)$ is Gamma function.

Equation (5) can be further decomposed as follows:

$$
D_{G L}^{\alpha} f(x)=\lim _{h \longrightarrow 0} \frac{1}{h^{\alpha}}\left(f(t)+(-\alpha) f(t-h)+\frac{(\alpha)(\alpha+1)}{2} f(t-h)+\cdots+\frac{\Gamma(\alpha+1)}{k ! \Gamma(\alpha-N+1)} f(t-j h)\right) .
$$

We know that equation (6) is the fractional derivative operator with $\alpha>0$, and it takes a part of the fractional integral operator with $\alpha<0$. When $\alpha>0$, we set $\gamma=-\alpha$, and the integral GL of order $\gamma$ using equation (6) is described as 


$$
J_{G L}^{\gamma} f(x) \approx \frac{1}{\Gamma(\gamma)} \int_{0}^{t} \frac{f(\tau)}{(t-\tau)^{1-\gamma}} \mathrm{d} \tau=\lim _{h \longrightarrow 0} \frac{1}{h^{-\gamma}}\left(f(t)+\gamma f(t-h)+\frac{(-\gamma)(-\gamma+1)}{2} f(t-h)+\cdots+\frac{\Gamma(-\gamma+1)}{k ! \Gamma(-\gamma-N+1)} f(t-j h)\right) .
$$

From the above discussion, the $\mathrm{ABC}$-fractional integral can be described by equation (7):

$$
\begin{aligned}
{ }_{0}^{A B C} J_{t}^{\beta}(t) & =\frac{1-\gamma}{A(\gamma)} f(t)+\frac{\gamma}{\Gamma(\gamma) A(\gamma)} \int_{0}^{T} f(\tau)(t-\tau)^{\gamma-1} \mathrm{~d} \tau \\
& \approx \frac{1-\gamma}{A(\gamma)} f(t)+\frac{\gamma}{A(\gamma)} h^{-\gamma}\left(f(t)+\gamma f(t-h)+\frac{(-\gamma)(-\gamma+1)}{2} f(t-h)+\cdots\right) .
\end{aligned}
$$

In [26], the newly defined fractional order integral is mentioned. It can be approximated to the following as $t=t_{n}$ :

$$
\begin{aligned}
{ }_{0}^{A B C} J_{t}^{\gamma}\left(t_{n}\right) & =\frac{1-\gamma}{A(\gamma)} f(t)+\frac{\gamma}{\Gamma(\gamma) A(\gamma)} \int_{0}^{t_{n}} f(\tau)(t-\tau)^{\gamma-1} \mathrm{~d} \tau \\
& =\frac{1-\gamma}{A(\gamma)} f(t)+\frac{\gamma}{\Gamma(\gamma) A(\gamma)} \sum_{k=0}^{N} \int_{t_{k}}^{t_{k+1}} f(\tau)(t-\tau)^{\gamma-1} \mathrm{~d} \tau .
\end{aligned}
$$

The function $f(\tau)$ can be described by a two-step Lagrange polynomial interpolation as follows:

$$
f(\tau)=\frac{f\left(t_{k}\right)}{h}\left(\tau-t_{k-1}\right)+\frac{f\left(t_{k-1}\right)}{h}\left(\tau-t_{k}\right) .
$$
follows:

Using equation (10), equation (9) can be discretized as

$$
\begin{aligned}
{ }_{0}^{A B C} J_{t}^{\gamma}\left(t_{n}\right)= & {\left[\frac{(1-\gamma) \Gamma(\gamma+2)+\gamma h^{\gamma}(\beta+2)}{A(\gamma) \Gamma(\gamma+2)}\right] f\left(t_{n}\right)+\left[\frac{\gamma h^{\gamma}}{A(\gamma)}\left(\frac{-2 * \gamma+(\gamma+3) 2^{\gamma}-4}{\Gamma(\gamma+2)}\right)\right] f\left(t_{n-1}\right) } \\
& +\left[\frac{\gamma h^{\gamma}\left(-2^{\gamma+1} \gamma+(\gamma+4) 3^{\gamma}+a-6 * 2^{\gamma}+2\right)}{A(\gamma) \Gamma(\gamma+2)}\right] f\left(t_{n-2}\right)+\cdots
\end{aligned}
$$

2.2. The Mask Based on Grunwald-Letnikov (GL_ABC) and Toufik-Atangana (TA-ABC). In an image, the distance between two pixels can be assumed to be 1 . This distance is the same as $h$ in equation (7). Therefore, the GL integral with fractional-order in the $x$ and $y$ directions [30] are described by equations (12) and (13):

$$
\begin{array}{ll}
{ }^{x} J_{G L}^{\gamma} f(x, y) \approx \frac{1}{A(\gamma)} f(x, y)+\frac{\gamma^{2}}{A(\gamma)} f(x-1, y)+\frac{\gamma^{3}-\gamma^{2}}{2 A(\gamma)} f(x-2, y)+\cdots, & (0<\gamma \leq 1), \\
{ }^{y} J_{G L}^{\gamma} f(x, y) \approx \frac{1}{A(\gamma)} f(x, y)+\frac{\gamma^{2}}{A(\gamma)} f(x, y-1)+\frac{\gamma^{3}-\gamma^{2}}{2 A(\gamma)} f(x, y-2)+\cdots, \quad(0<\gamma \leq 1) .
\end{array}
$$


The TA_ABC integral with fractional-order [31] in the $x$ and $y$ directions is presented as equations (14) and (15):

$$
\begin{aligned}
{ }^{x} J_{\mathrm{TA}-\mathrm{ABC}}^{\gamma} f(x, y) \approx & {\left[\frac{(1-\gamma) \Gamma(\gamma+2)+\gamma(\gamma+2)}{A(\gamma) \Gamma(\gamma+2)}\right] f(x, y) } \\
& +\left[\frac{\gamma h^{\gamma}}{A(\gamma)}\left(\frac{-2 * \gamma+(\gamma+3) 2^{\gamma}-4}{\Gamma(\gamma+2)}\right)\right] f(x-1, y)+\left[\frac{\gamma\left(-2^{\gamma+1} \gamma+(\gamma+4) 3^{\gamma}+a-6 * 2^{\gamma}+2\right)}{A(\gamma) \Gamma(\gamma+2)}\right] f(x-2, y), \\
{ }^{y} J_{\mathrm{TA}-\mathrm{ABC}}^{\gamma} f(x, y) \approx & {\left[\frac{(1-\gamma) \Gamma(\gamma+2)+\gamma(\gamma+2)}{A(\gamma) \Gamma(\gamma+2)}\right] f(x, y) } \\
& +\left[\frac{\gamma h^{\gamma}}{A(\gamma)}\left(\frac{-2 * \gamma+(\gamma+3) 2^{\gamma}-4}{\Gamma(\gamma+2)}\right)\right] f(x, y-1)+\left[\frac{\gamma\left(-2^{\gamma+1} \gamma+(\gamma+4) 3^{\gamma}+a-6 * 2^{\gamma}+2\right)}{A(\gamma) \Gamma(\gamma+2)}\right] f(x, y-2) .
\end{aligned}
$$

with equations $(12)-(15)$, the $5 * 5$ fractional integral mask can be constructed as follows:

This $5 * 5$ mask is used in image denoising as the filter. The mask is rotation-invariant mainly because it is obtained by superimposition of fractional integral in eight directions. Thus, we can use different fractional-order integrals for airspace filtering to denoise images. Therefore, the coefficients of GL_ABC and TA_ABC mask are described by equations (16) and (17), respectively (Table 1).

$$
\begin{aligned}
& \mathrm{H}_{0}=\frac{1}{A(\gamma)}, \\
& \mathrm{H}_{1}=\frac{\gamma^{2}}{A(\gamma)}, \\
& \mathrm{H}_{2}=\frac{\gamma^{3}-\gamma^{2}}{2 A(\beta)}, \\
& \mathrm{H}_{0}=\frac{(1-\gamma) \Gamma(\gamma+2)+\gamma(\gamma+2)}{A(\gamma) \Gamma(\gamma+2)}, \\
& \mathrm{H}_{1}=\frac{\gamma}{A(\gamma)}\left(\frac{-2 \gamma+(\gamma+3) 2^{\gamma}-4}{\Gamma(\gamma+2)}\right), \\
& \mathrm{H}_{2}=\frac{\gamma\left(-2^{\gamma+1} \gamma+(\gamma+4) 3^{\gamma}+a-6 * 2^{\gamma}+2\right)}{A(\gamma) \Gamma(\gamma+2)} .
\end{aligned}
$$

\section{Adaptive Fractional Operators Based on Atangana-Baleanu Derivatives}

For an image with noise of different intensities and in different regions, one fixed order in the fractional integral operator is insufficient to achieve a good denoising effect. Therefore, this paper proposes an adaptive fractional operator for image denoising. The edge intensity coefficient, image entropy, local entropy, and local variance weight are used to construct the expression of the adaptive fractional order. The image gradient represents the image edge intensity information. In this paper, The Kirsch algorithm is applied to calculate the image edge intensity. However, the Kirsch algorithm can suppress image noise [32].

$$
G[I(x, y)]=\max \left\{1, \max \left\{\left|5 r_{k}-3 s_{k}\right|, \quad k=0,1, \ldots, 7\right\}\right\} .
$$

Here, $\quad r_{k}=W_{k}+W_{k-(\pi / 4)}+W_{k-(\pi / 2)} ; s_{k}=W_{k-(3 \pi / 4)}+$ $W_{k-\pi}+W_{k-(5 \pi / 4)}+W_{k-(3 \pi / 2)}+W_{k-(7 \pi / 4)}$. Moreover, when $k=(0,(\pi / 4),(\pi / 2),(3 \pi / 4), \pi,(5 \pi / 4),(3 \pi / 2),(7 \pi / 4))$, the eight directions of masks are depicted as follows:

$$
\begin{aligned}
W_{0} & =\left[\begin{array}{ccc}
-3 & -3 & 5 \\
-3 & 0 & 5 \\
-3 & -3 & 5
\end{array}\right], \\
W_{(\pi / 4)} & =\left[\begin{array}{ccc}
-3 & 5 & 5 \\
-3 & 0 & 5 \\
-3 & -3 & -3
\end{array}\right], \\
W_{(\pi / 2)} & =\left[\begin{array}{ccc}
5 & 5 & 5 \\
-3 & 0 & -3 \\
-3 & -3 & -3
\end{array}\right], \\
W_{(3 \pi / 4)} & =\left[\begin{array}{ccc}
5 & 5 & -3 \\
5 & 0 & -3 \\
-3 & -3 & -3
\end{array}\right], \\
W_{(5 \pi / 4)} & =\left[\begin{array}{ccc}
5 & -3 & -3 \\
5 & 0 & -3 \\
5 & 0 & -3 \\
5 & 5 & -3
\end{array}\right],
\end{aligned}
$$


TABLE 1: $5^{*} 5$ mask.

\begin{tabular}{lcccc}
\hline $\mathrm{H}_{2}$ & 0 & $\mathrm{H}_{2}$ & 0 & $\mathrm{H}_{2}$ \\
0 & $\mathrm{H}_{1}$ & $\mathrm{H}_{1}$ & $\mathrm{H}_{1}$ & 0 \\
$\mathrm{H}_{2}$ & $\mathrm{H}_{1}$ & $8 \mathrm{H}_{0}$ & $\mathrm{H}_{1}$ & $\mathrm{H}_{2}$ \\
0 & $\mathrm{H}_{1}$ & $\mathrm{H}_{1}$ & $\mathrm{H}_{1}$ & 0 \\
$\mathrm{H}_{2}$ & 0 & $\mathrm{H}_{2}$ & 0 & $\mathrm{H}_{2}$ \\
\hline
\end{tabular}

$$
\begin{aligned}
& W_{(3 \pi / 2)}=\left[\begin{array}{ccc}
-3 & -3 & -3 \\
-3 & 0 & -3 \\
5 & 5 & 5
\end{array}\right], \\
& W_{(7 \pi / 4)}=\left[\begin{array}{ccc}
-3 & -3 & -3 \\
-3 & 0 & 5 \\
-3 & 5 & 5
\end{array}\right] .
\end{aligned}
$$

Image entropy determines how much information an image contains. The smaller the entropy is, the more information it contains [31].

$$
E_{l}=-\sum_{L=1}^{255} P\left(I_{j, k}\right) \log _{2} P\left(I_{j, k}\right) \text {, }
$$

$P$ is the probability that an image pixel will appear. The local variance weight can not only measure the local gray change of an image but also reflect the importance of the image local change rate in the whole image. The larger the difference in partial pixel values is, the greater the local variance weight. Conversely, the smaller the changes are, the smaller the value of the local variance weight [33].

$$
\operatorname{St}(h)=\frac{1}{\operatorname{Num}} \sum_{i=1}^{\mathrm{Num}} \frac{\sigma_{I}^{2}(h \prime)}{\sigma_{I}^{2}(h)},
$$

where Num represents the number of image pixels, $I$ is the image to be processed, $h$ is the local pixel, $h$ ' is the local pixel of the current window, and $\sigma_{I}^{2}\left(h^{\prime}\right)$ is the variance in the pixel value in the current window.

The function established in this paper takes the global entropy of the image as a measure of the overall image characteristics. The order value should be small to maintain the texture details. We consider taking the product of three measures of frequency information, to ensure that the fractional-order is inversely proportional to high frequency information such as edges and texture details. The adaptive order function is as follows:

$$
\text { a } d a_{v}=E_{t} * \varepsilon-G * S t * E_{l},
$$

where $E_{t}$ represents the entropy of the whole image, $\varepsilon$ is the coefficient of $E_{t}$ and take 0.22 in the experiment, and $G * S t * E_{l}$ is the product of the local information entropy, local gradient, and local variance. Then, the entropy of the global image is subtracted from the product. The results are small in the region of the edge and texture and high in the region of smoothness. According to this equation, the orders of different local textures of the image vary. As shown in Figure 1, the order used in the edge and texture details is relatively small, while the order used in the smooth area is larger. In this way, the obtained orders are reduced in the edge and texture detail region and enhanced in the smooth region so that the edge and texture detail information can be preserved as much as possible while denoising.

\section{Numerical Examples}

In this paper, the peak signal-to-noise ratio (PSNR), entropy, and structural similarity index measurement (SSIM) are used to assess the performance of the proposed operator. The PSNR is the most popular assessment criterion to evaluate the performance of denoising algorithms. In general, the value of the PSNR is higher when the image quality is better. The PSNR is defined as follows [34]:

$$
\begin{aligned}
\text { PSNR } & =10 \lg \frac{255^{2}}{\mathrm{MSE}}, \\
\mathrm{MSE} & =\frac{1}{M * N} \sum_{j=1}^{M} \sum_{k=1}^{N}[I \prime(j, k)-I(j, k)]^{2},
\end{aligned}
$$

where $M$ and $N$ are the size dimensions of the original image. $I(j, k)$ and $I^{\prime}(j, k)$ are the original and denoised images, respectively. The SSIM is also a well-known criterion among image quality assessment metrics [35] defined as

$$
\operatorname{SSIM}=\frac{\left(2 \varphi_{p} \varphi_{q}+\rho_{1}\right)\left(2 \sigma_{p q}+\rho_{2}\right)}{\left(\varphi_{p}^{2}+\varphi_{q}^{2}+\rho_{1}\right)\left(\sigma_{p}^{2}+\sigma_{b}^{2}+\rho_{2}\right)},
$$

where $p$ and $q$ represent different images; $\varphi_{p}$ and $\varphi_{q}$ represent the mean of images $p$ and $q ; \sigma_{p}^{2}$ and $\sigma_{b}^{2}$ represent the variances of $p$ and $q$, respectively; $\sigma_{p q}$ is the covariance of $p$ and $q$; and $\rho_{1}$ and $\rho_{2}$ are constants added to maintain stability. The value of the SSIM represents how similar two images are. When the SSIM value is higher, the pixel values of the two images are closer. The range of this index is $[0,1]$. If the value of this index is closer to one, the two images are more approximate.

In the experiments, we employed five grayscale images to test the proposed mask: "Lena," "Elaine," "Goldhill," "Peppers," and "Cameraman," with $512 * 512$ pixels each. We use the proposed adaptive function to improve the TA_ABC and GL_ABC mask. The improved mask "Ada_TA_ABC" and "Ada_GL_ABC" compare the "GL_ABC mask" [31], “TA_ABC mask” [30], and the method proposed in [36]. The orders $\gamma$ in "GL_ABC mask" and "TA_ABC mask" are from literature [30]. In the experiments, we added noise with different variances $\sigma \in\{15,20,25\}$ to the test images, respectively. Figures $2-16$ show the results for image denoising by the different methods. Tables 2-7 show the PSNR, SSIM and entropy of these test images for the different algorithms. From Figures 1-16, we find that the test images lost image details when TA_ABC mask was applied. The method proposed by [36] has a poor denoising ability. The Ada_TA_ABC mask performs better than the TA_ABC mask, the order of which is determined by our proposed 


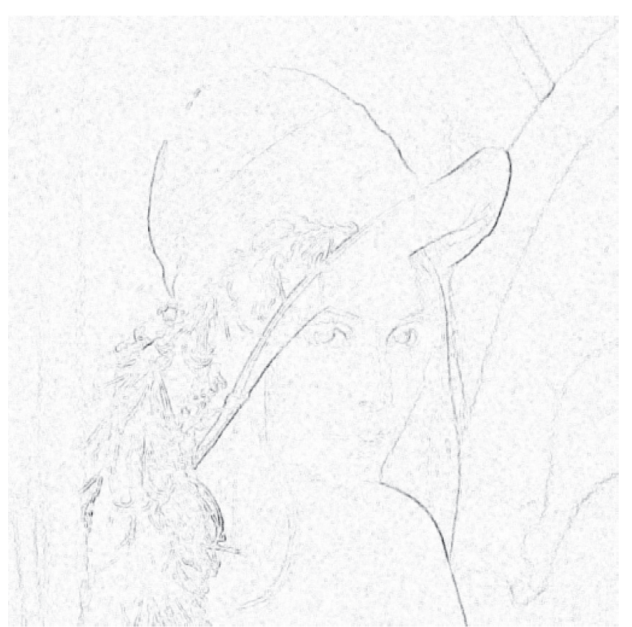

(a)

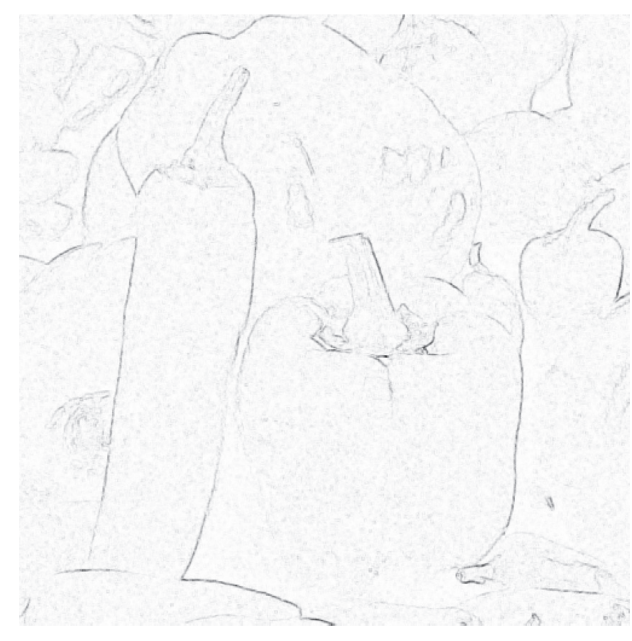

(b)

Figure 1: A map of the adaptive order. (a) Lena. (b) Peppers.

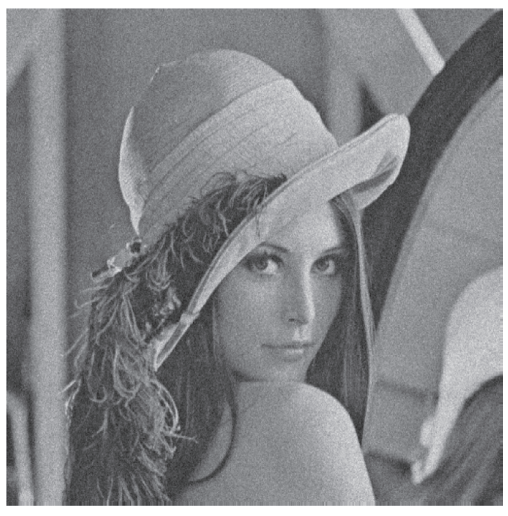

(a)

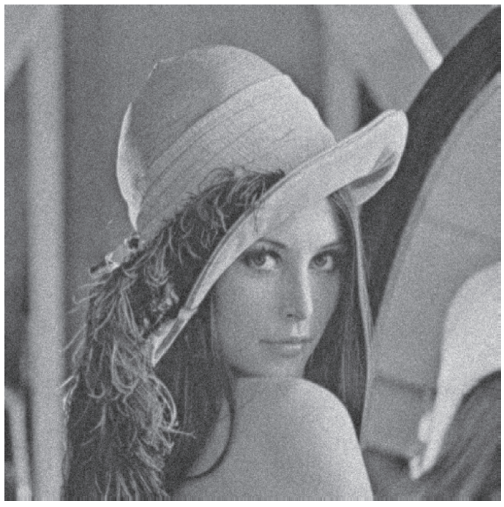

(d)

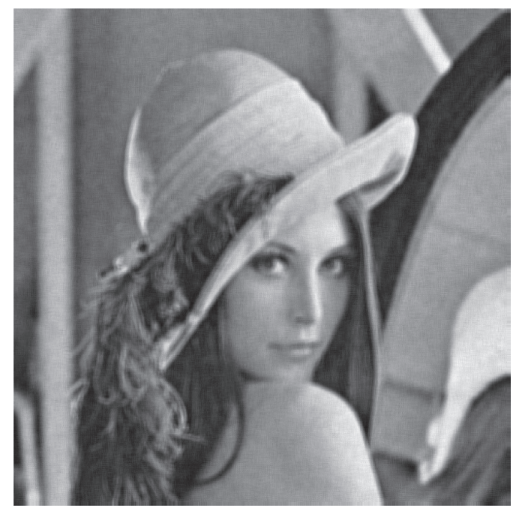

(b)

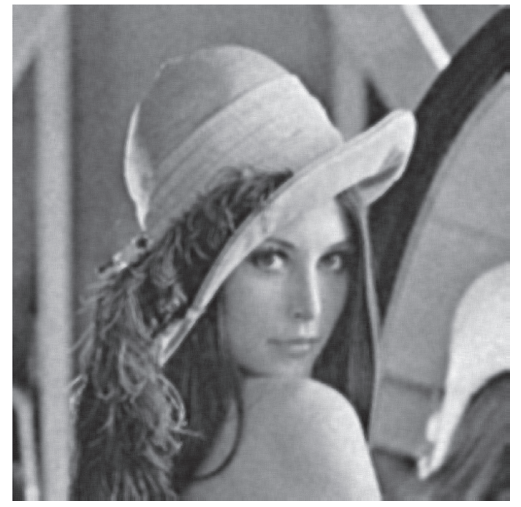

(e)

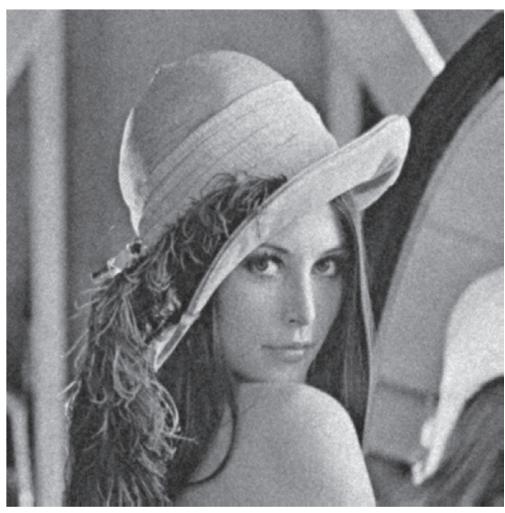

(c)

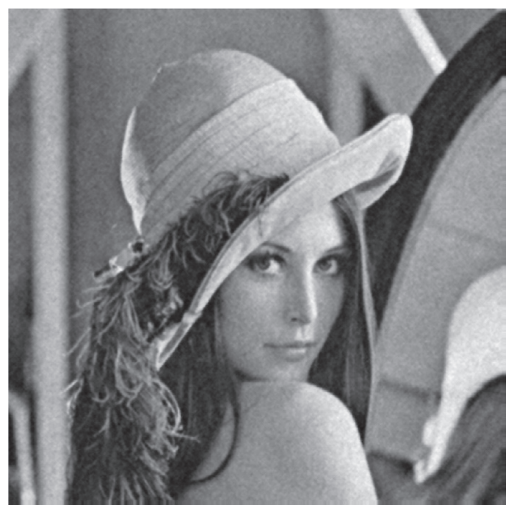

(f)

Figure 2: Comparison of different operators on "Lena" under Gaussian noise with variance $\sigma=15$.

method, as depicted by equation (15). The test images processed by the GL_ABC mask and Ada_GL_ABC mask contain less noise and more details. The Ada_GL_ABC mask is better than the GL_ABC mask. From another perspective, this result shows that the adaptive function proposed by us has certain universality. This result is verified in Tables 2-6. The PNSR of images proposed by the Ada_GL_ABC mask is higher than that of the other methods. This outcome means that the quality of images processed by the Ada_GL_ABC mask is better than that delivered by other methods. Meanwhile, the images processed by the Ada_GL_ABC mask are closer to the original images. This conclusion can be confirmed by the higher SSIM, which indicates the similarity between two images. Additionally, we calculated 


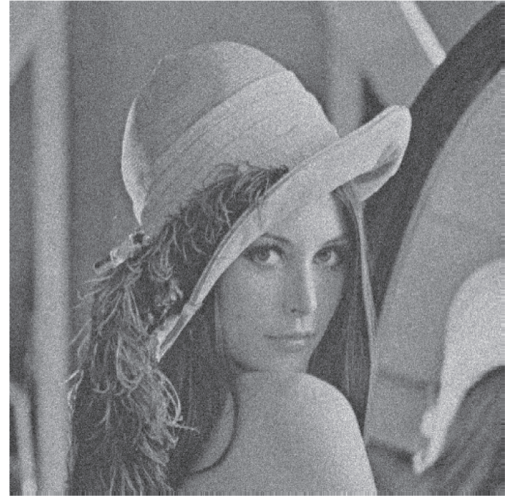

(a)

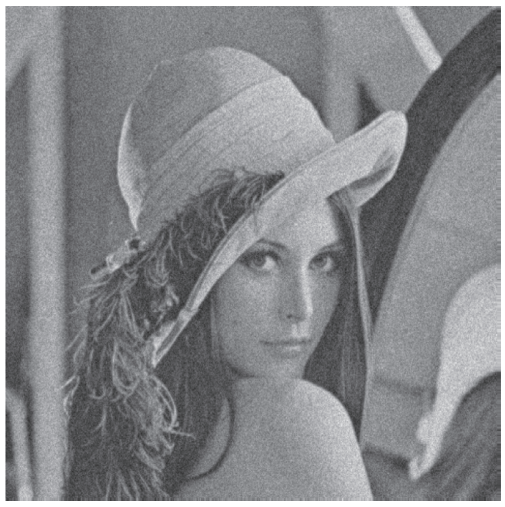

(d)

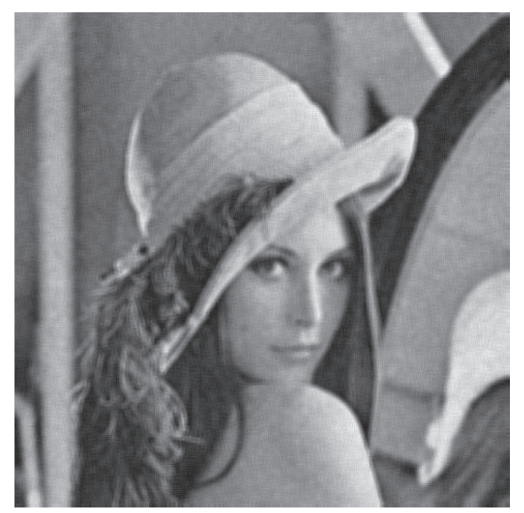

(b)

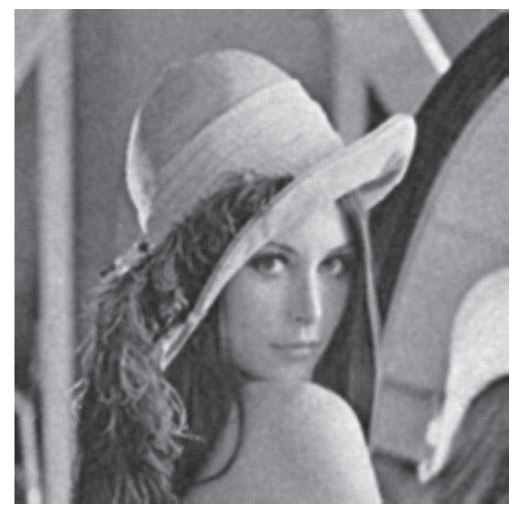

(e)

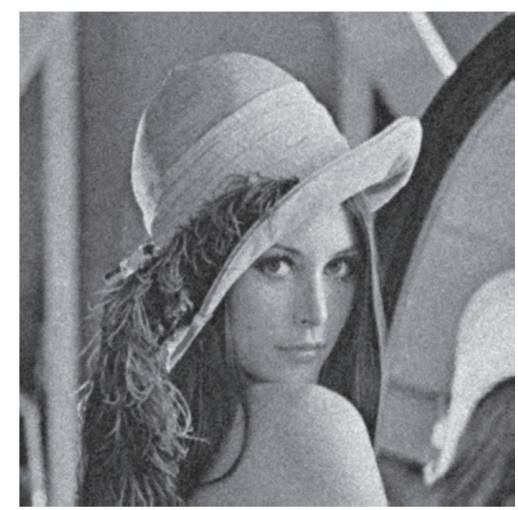

(c)

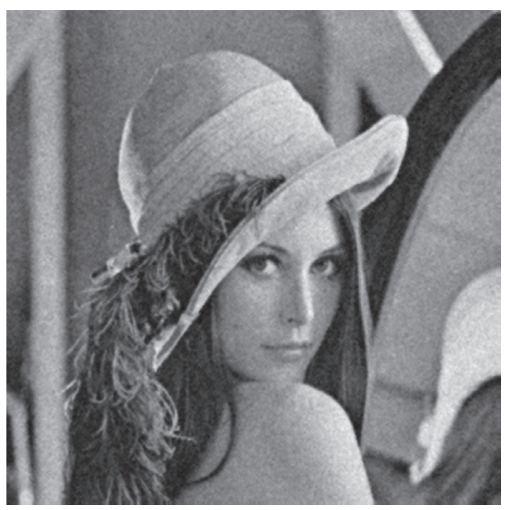

(f)

Figure 3: Comparison of different operators on "Lena" under Gaussian noise with variance $\sigma=20$.

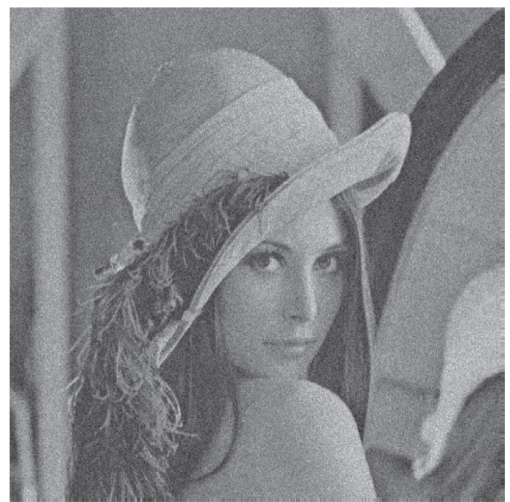

(a)

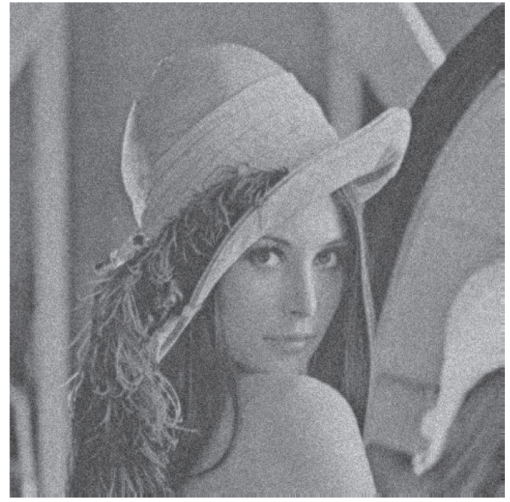

(d)

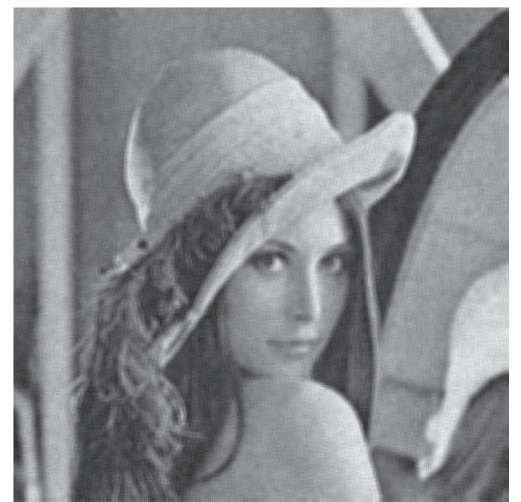

(b)

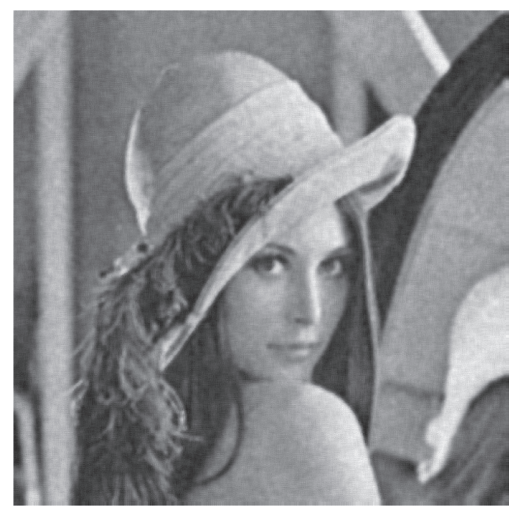

(e)

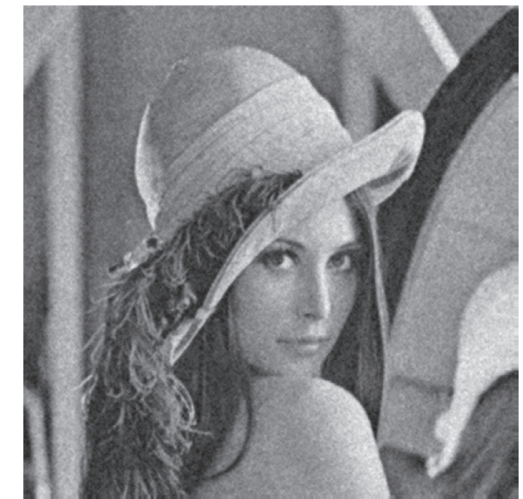

(c)

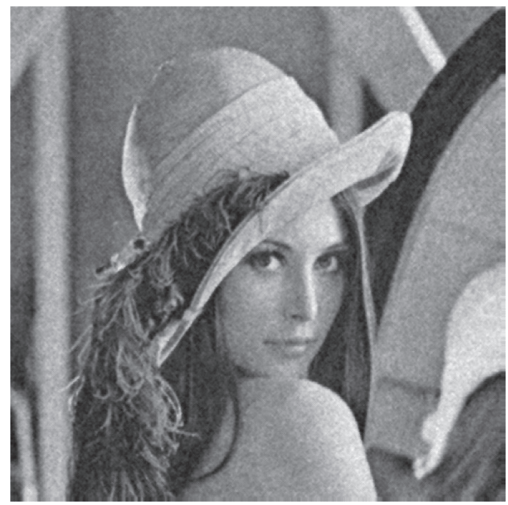

(f)

FIGURE 4: Comparison of different operators on "Lena" under Gaussian noise with variance $\sigma=25$. 


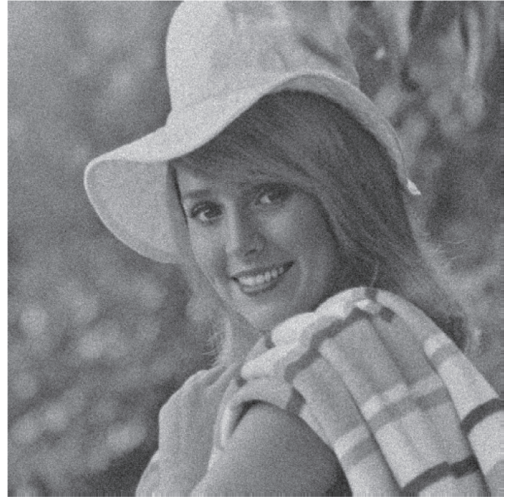

(a)

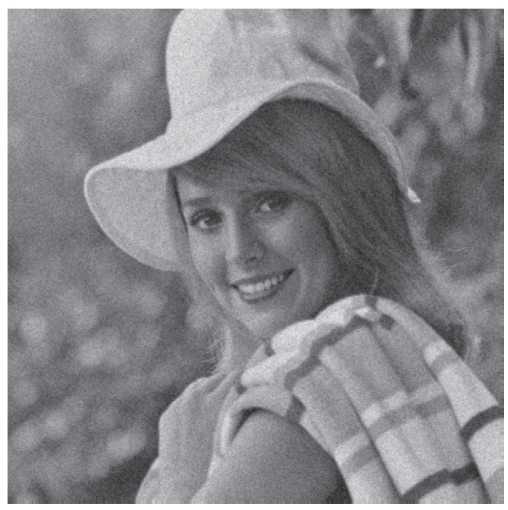

(d)

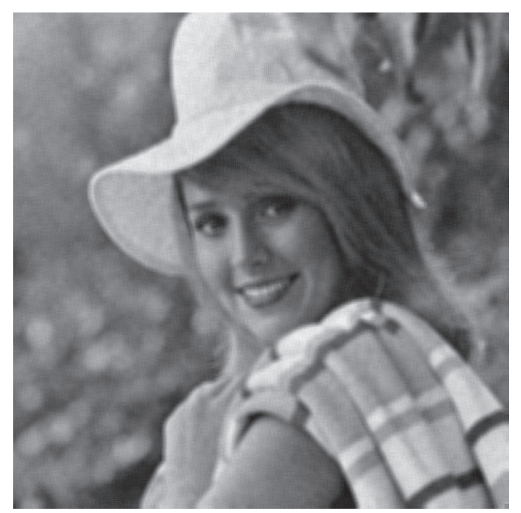

(b)

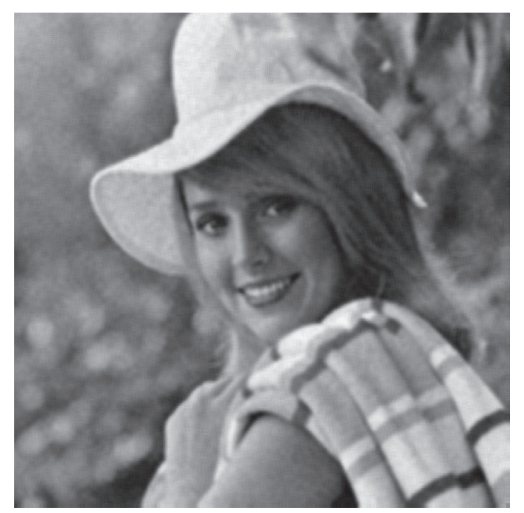

(e)

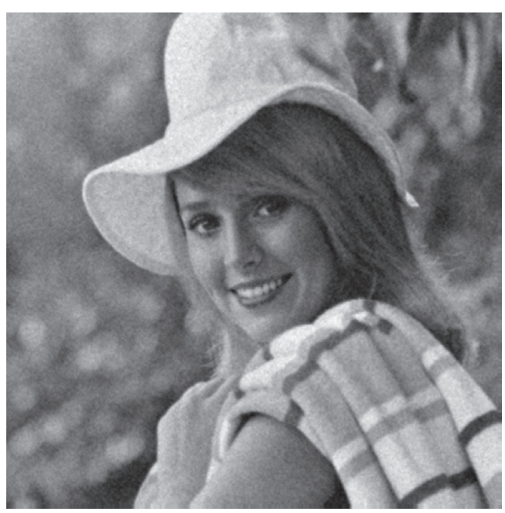

(c)

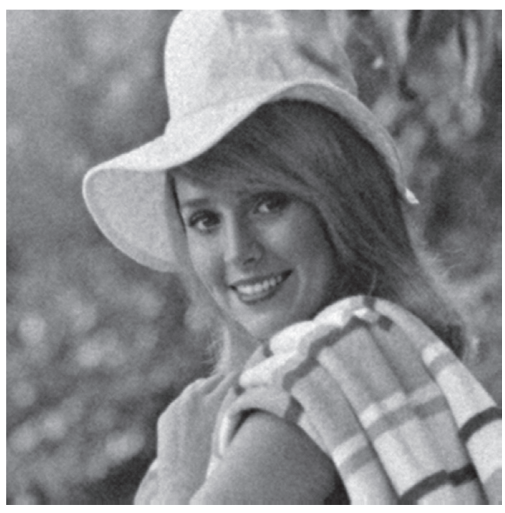

(f)

FiguRE 5: Comparison of different operators on "Elaine" under Gaussian noise with variance $\sigma=15$.

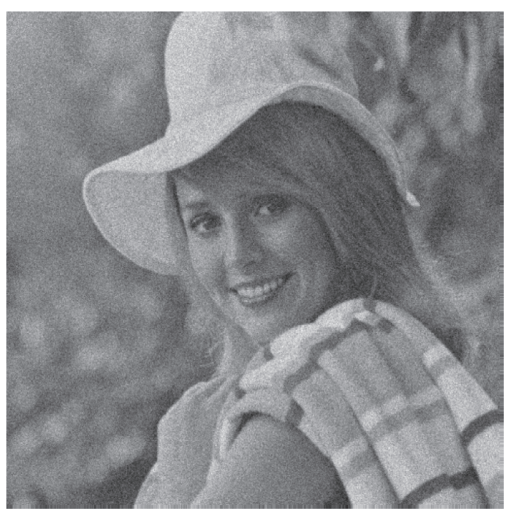

(a)

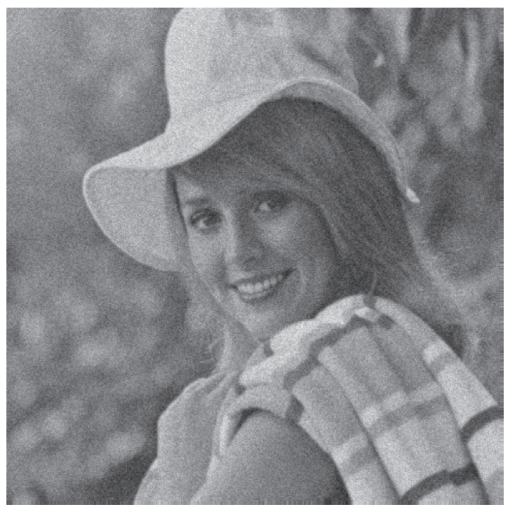

(d)

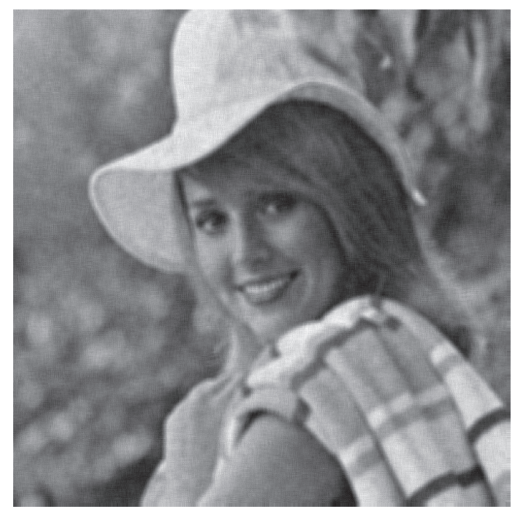

(b)

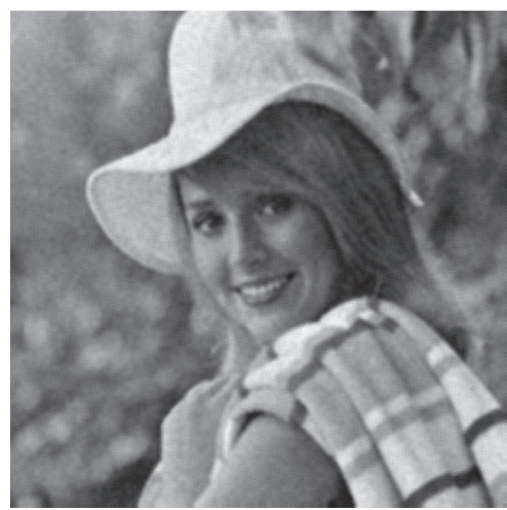

(e)

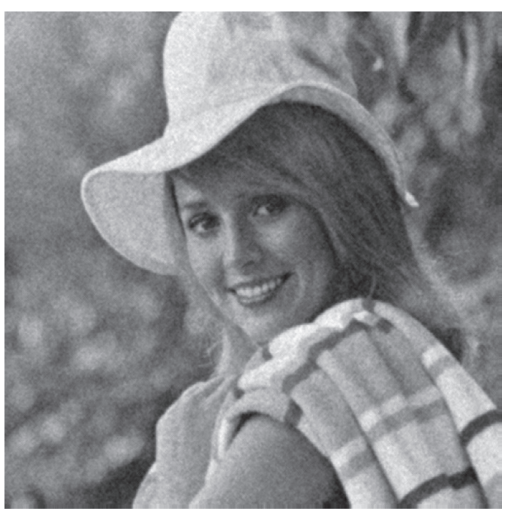

(c)

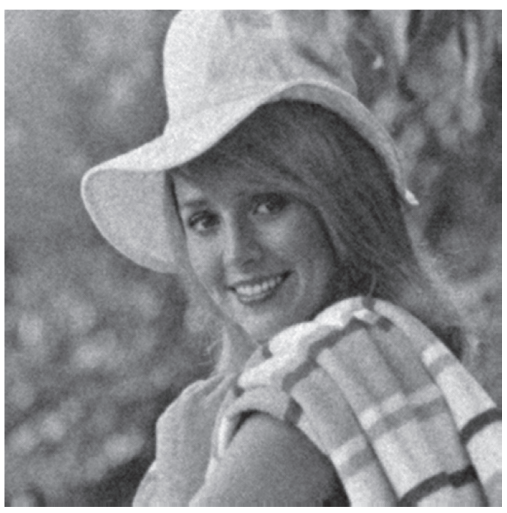

(f)

Figure 6: Comparison of different operators on "Elaine" under Gaussian noise with variance $\sigma=20$. 


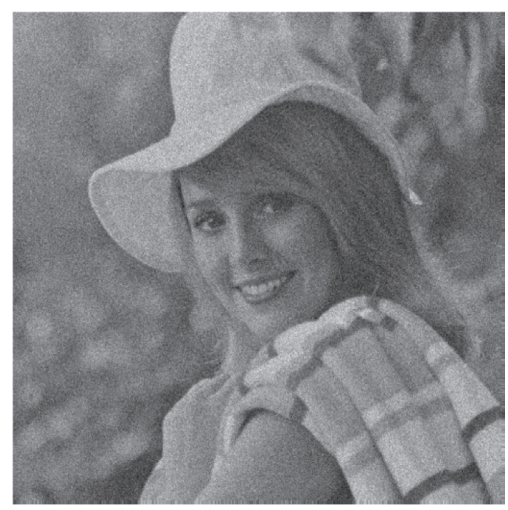

(a)

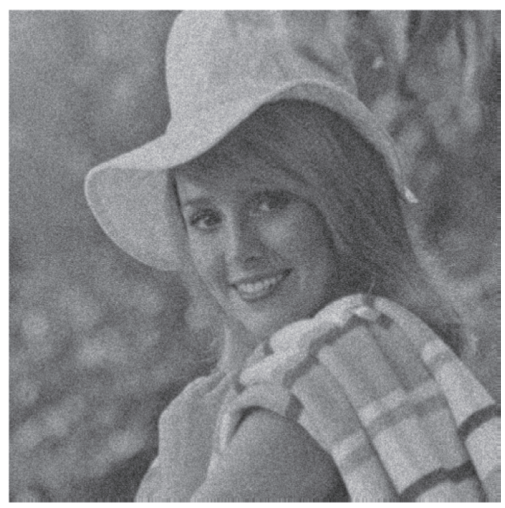

(d)

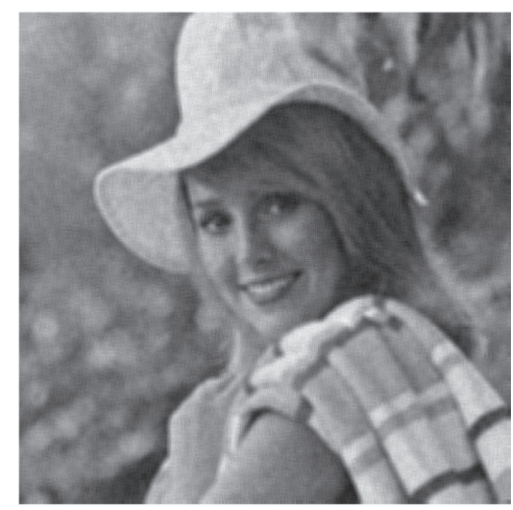

(b)

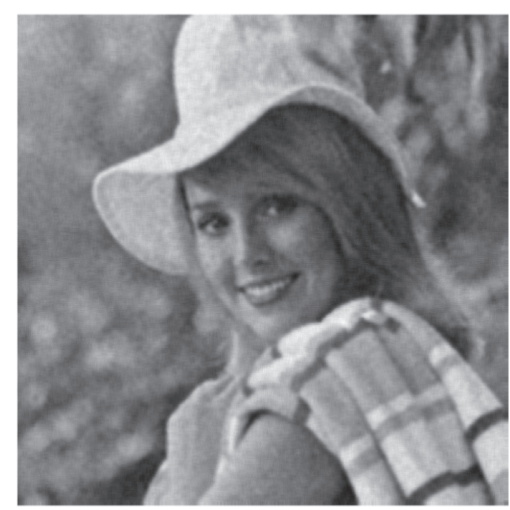

(e)

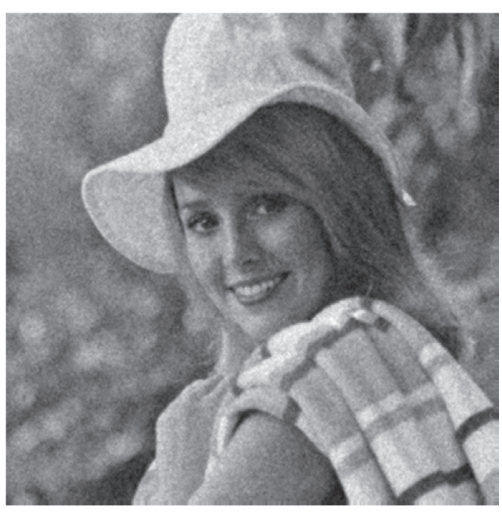

(c)

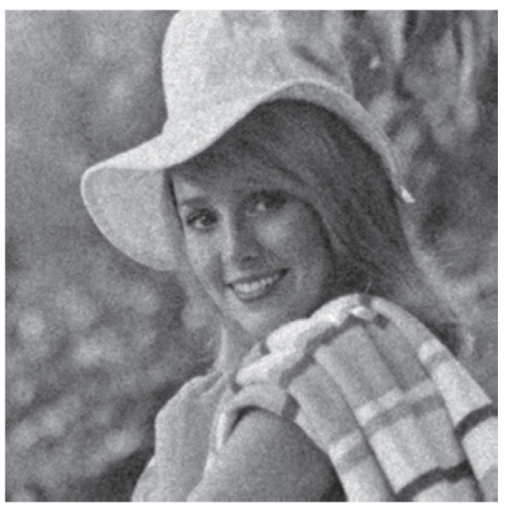

(f)

FIgURE 7: Comparison of different operators on "Elaine" under Gaussian noise with variance $\sigma=25$.

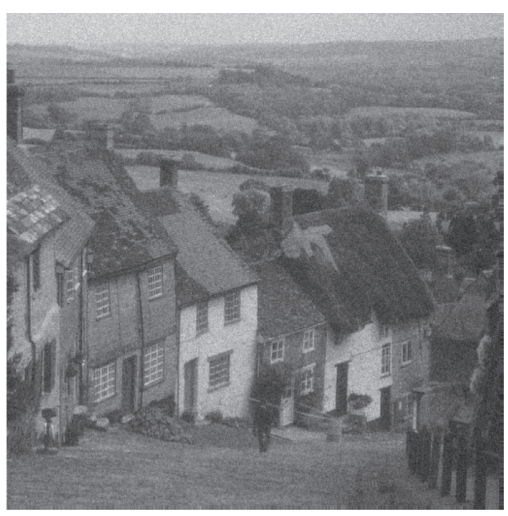

(a)

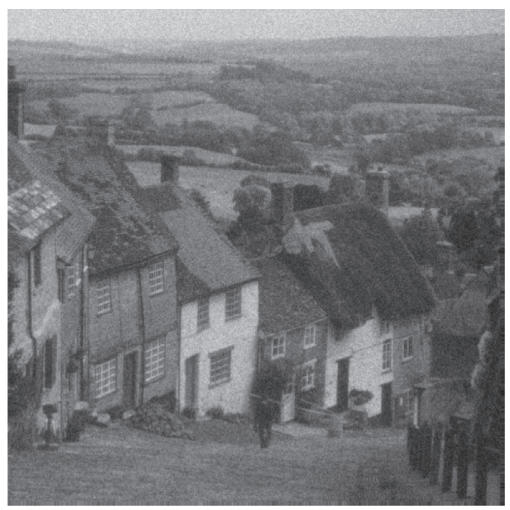

(d)

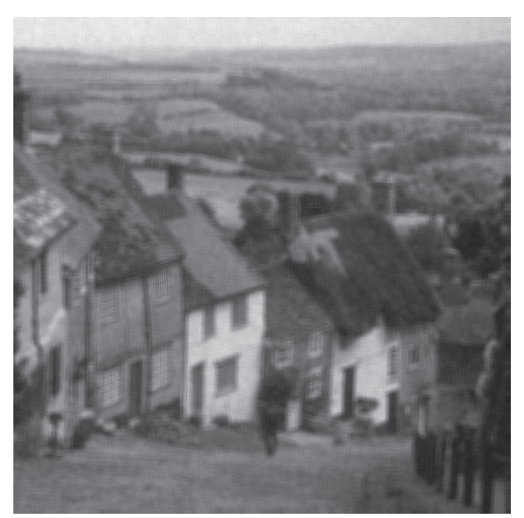

(b)

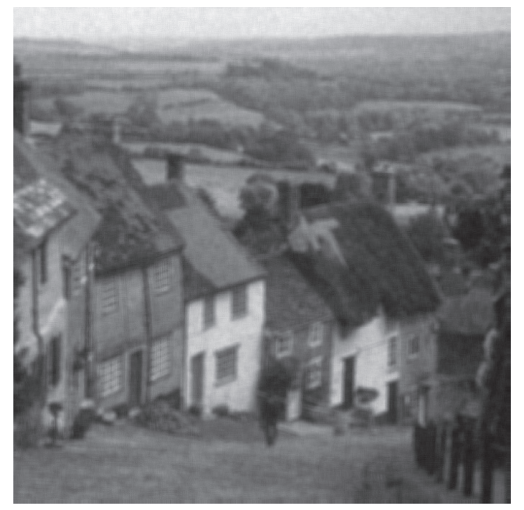

(e)

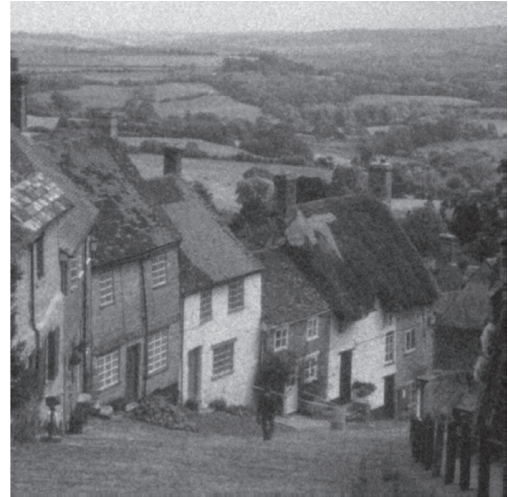

(c)

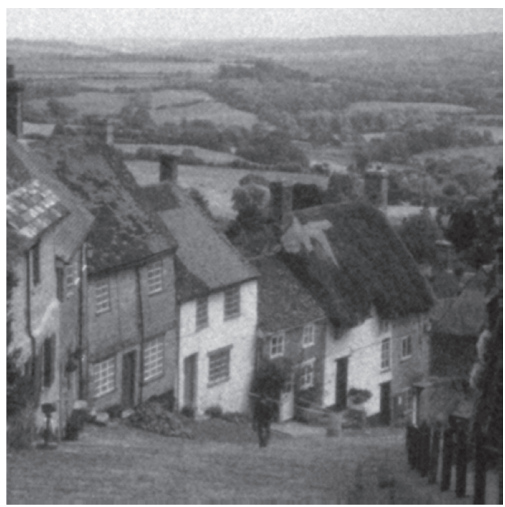

(f)

Figure 8: Comparison of different operators on "Goldhill” under Gaussian noise with variance $\sigma=15$. 


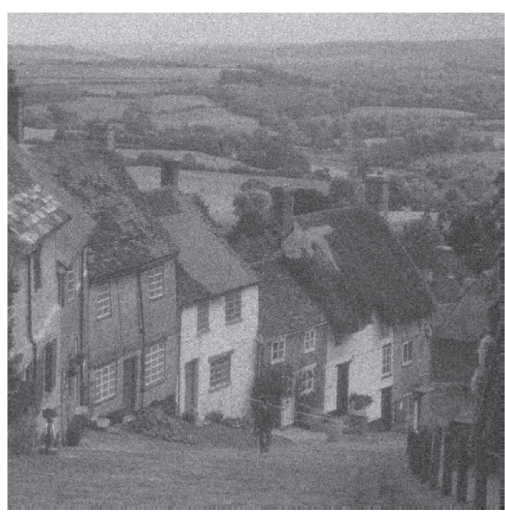

(a)

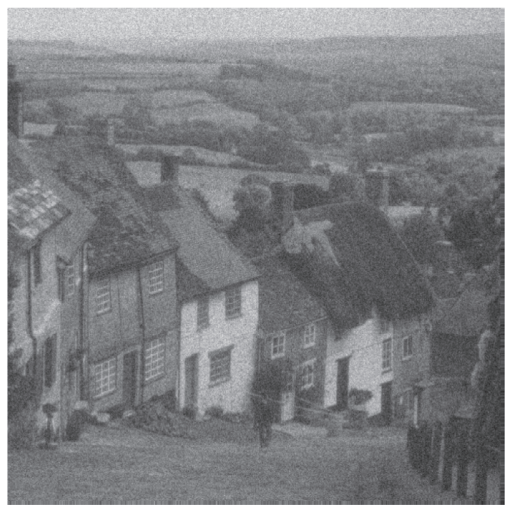

(d)

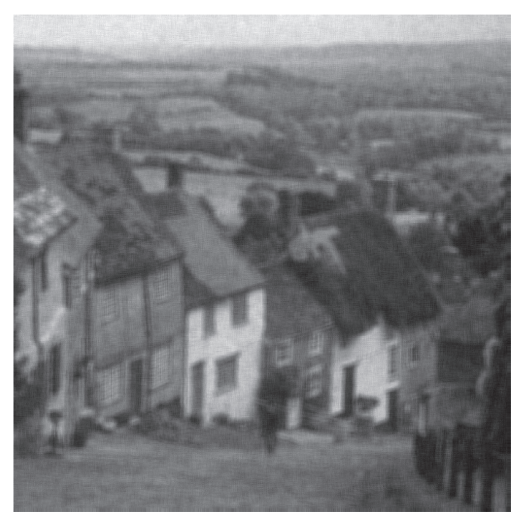

(b)

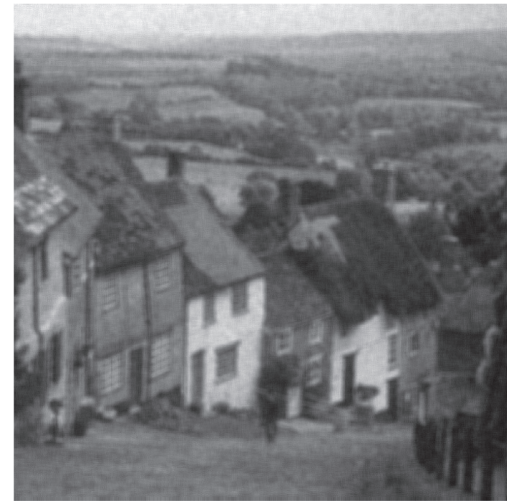

(e)

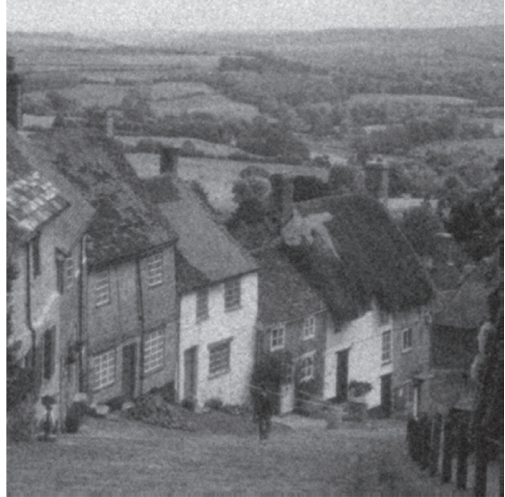

(c)

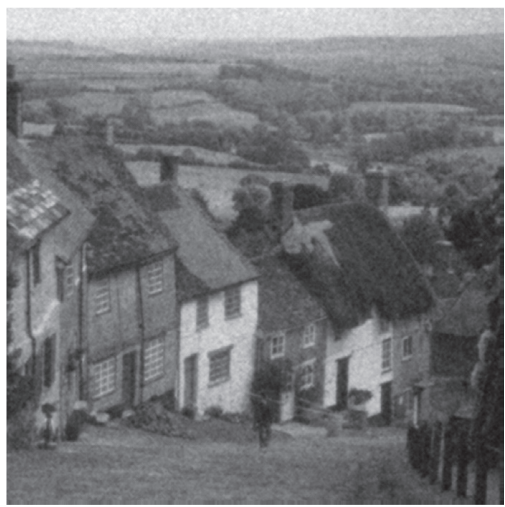

(f)

Figure 9: Comparison of different operators on "Goldhill" under Gaussian noise with variance $\sigma=20$.

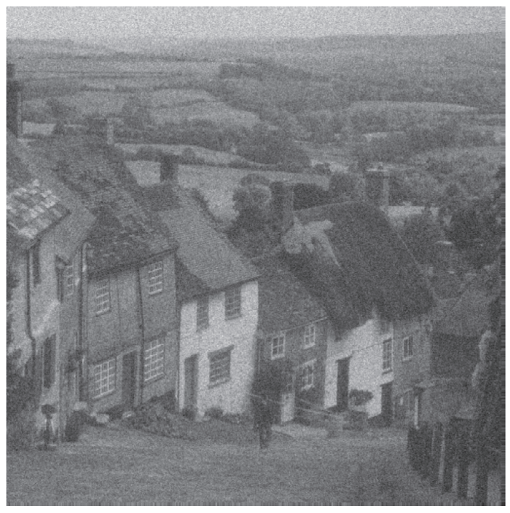

(a)

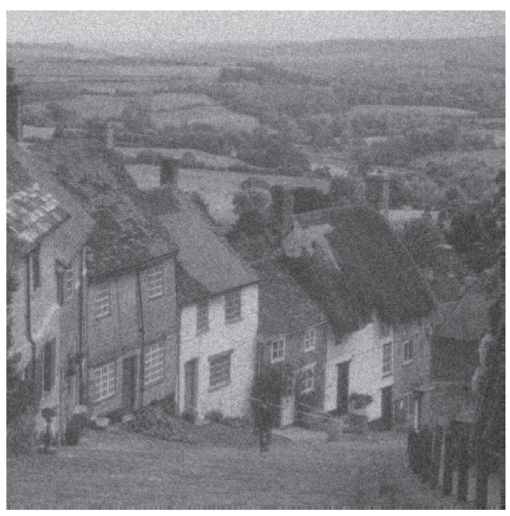

(d)

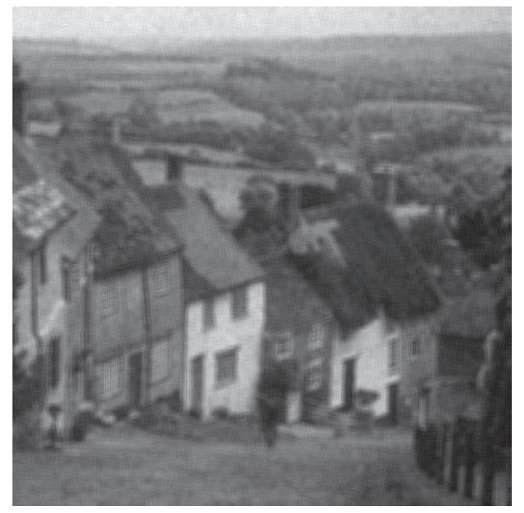

(b)

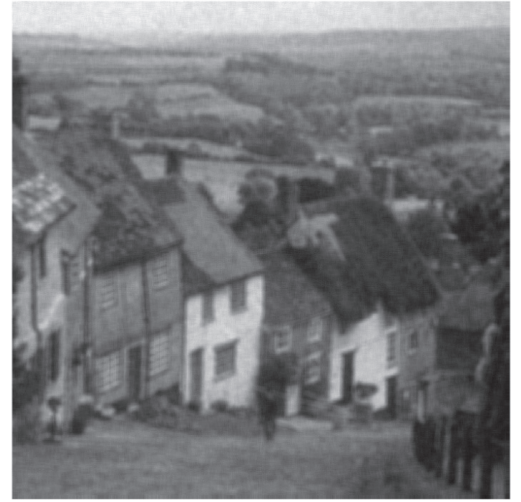

(e)

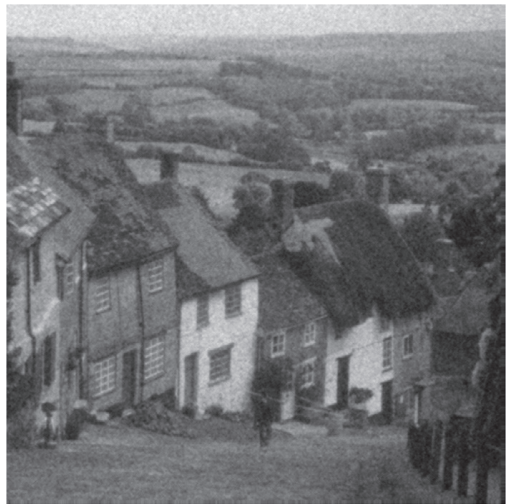

(c)

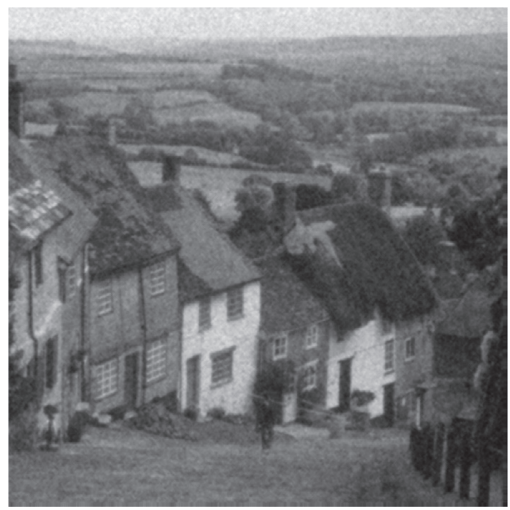

(f)

Figure 10: Comparison of different operators on "Goldhill" under Gaussian noise with variance $\sigma=25$. 


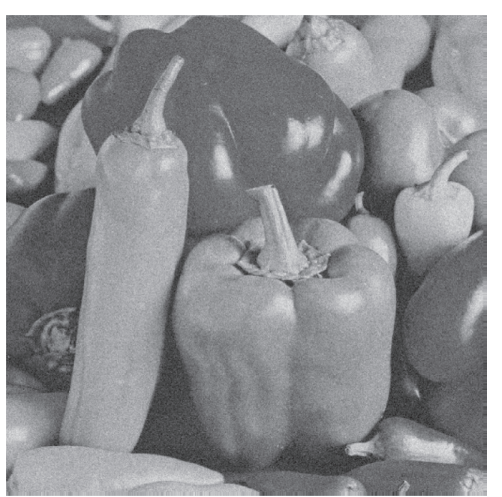

(a)

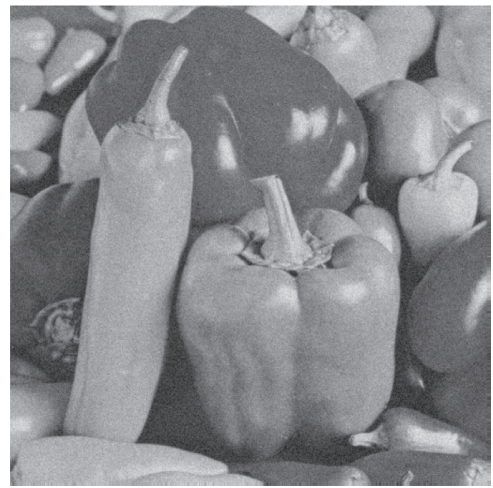

(d)

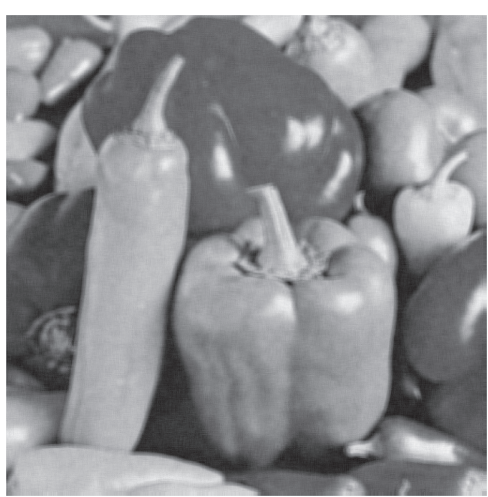

(b)

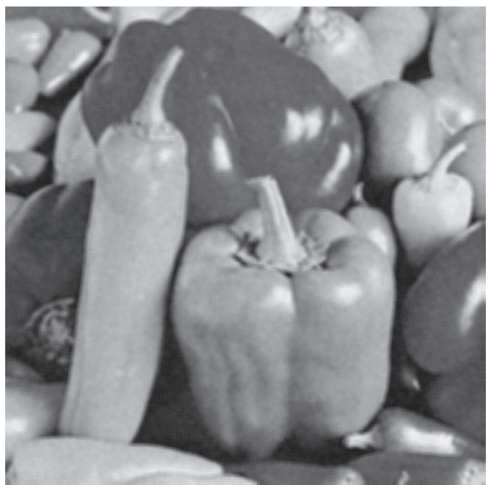

(e)

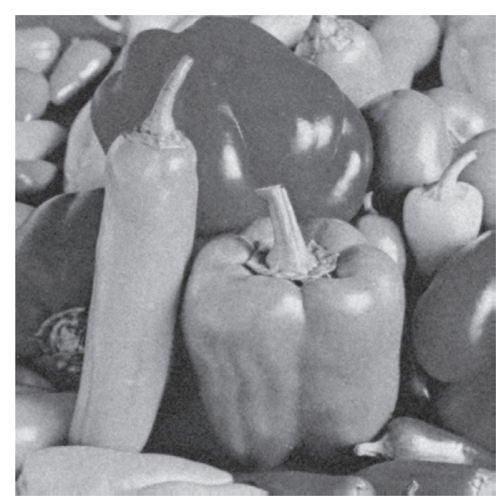

(c)

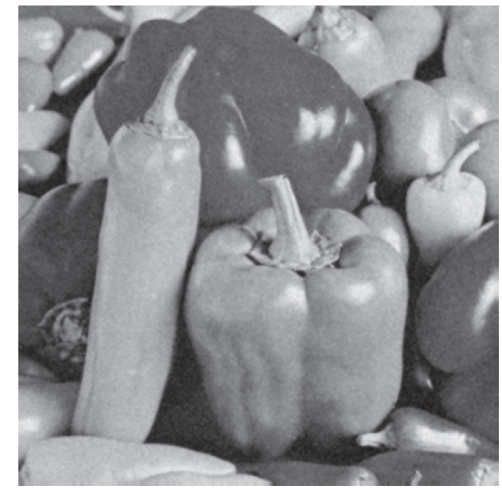

(f)

FIGURE 11: Comparison of different operators on "Pepper" under Gaussian noise with variance $\sigma=15$.

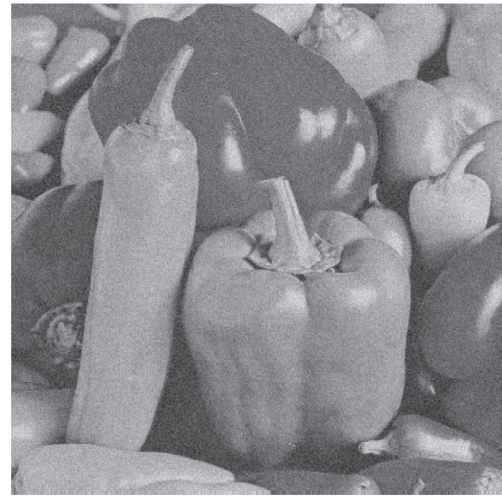

(a)

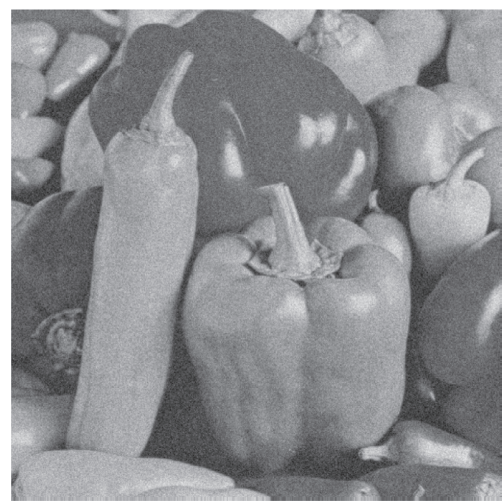

(d)

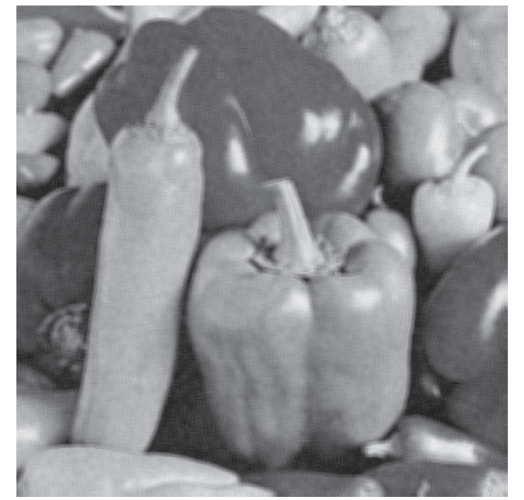

(b)

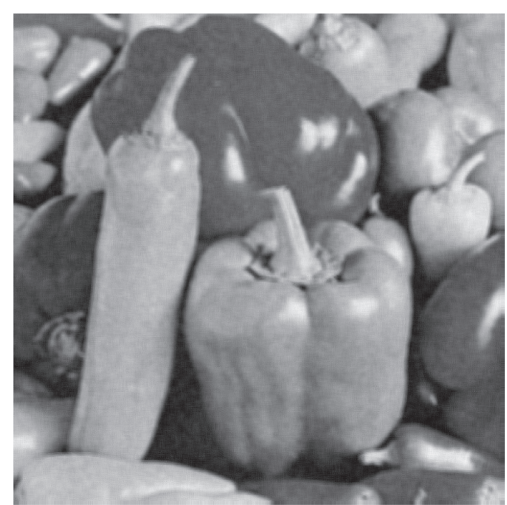

(e)

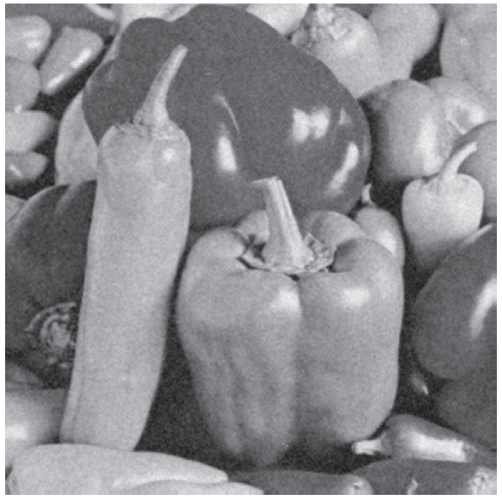

(c)

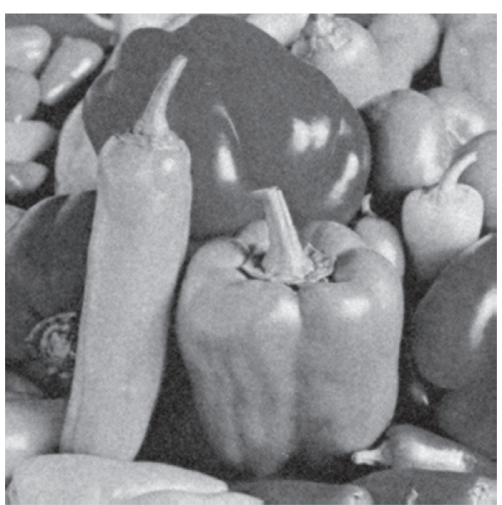

(f)

Figure 12: Comparison of different operators on "Pepper" under Gaussian noise with variance $\sigma=20$. 


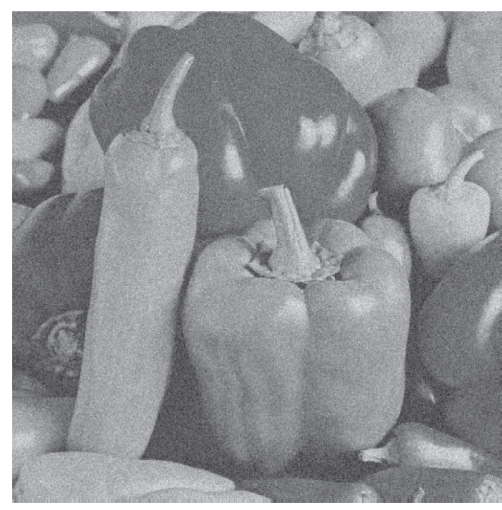

(a)

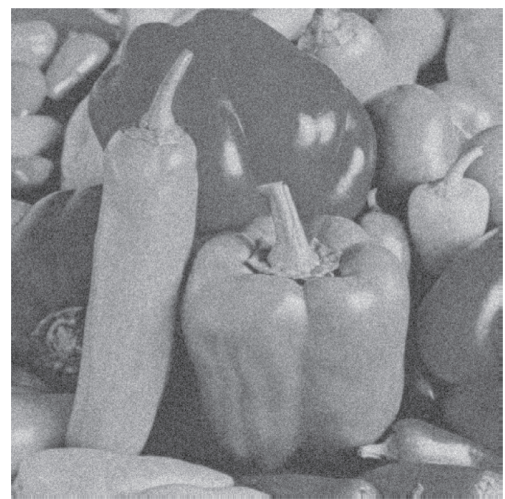

(d)

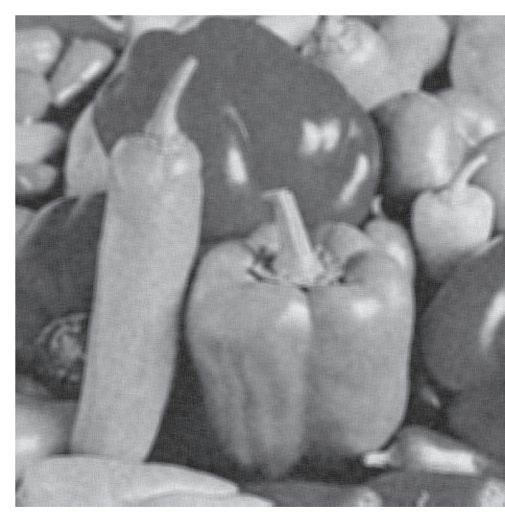

(b)

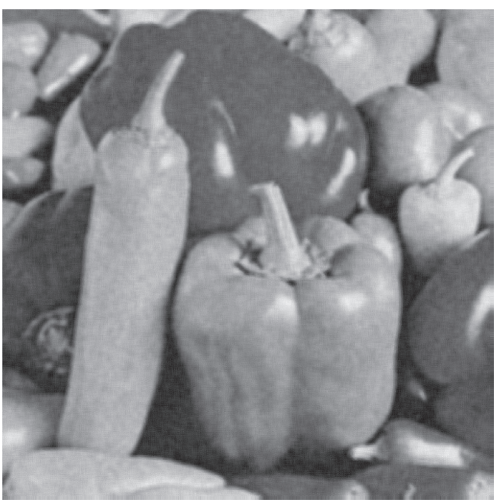

(e)

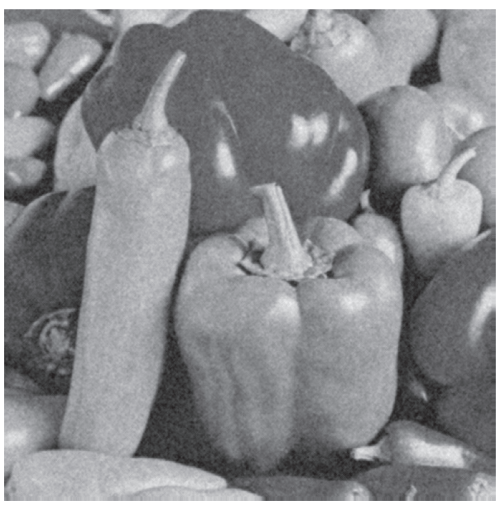

(c)

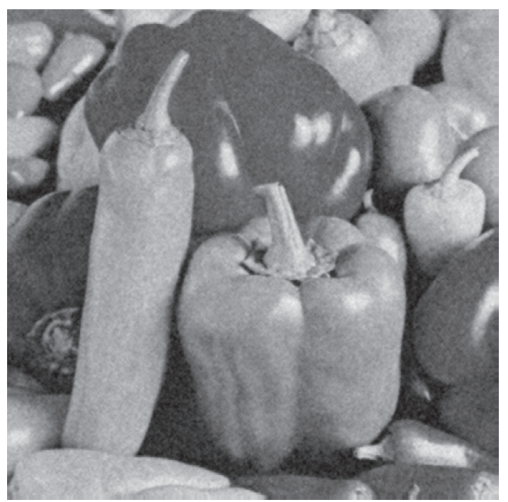

(f)

Figure 13: Comparison of different operators on "Pepper" under Gaussian noise with variance $\sigma=25$.

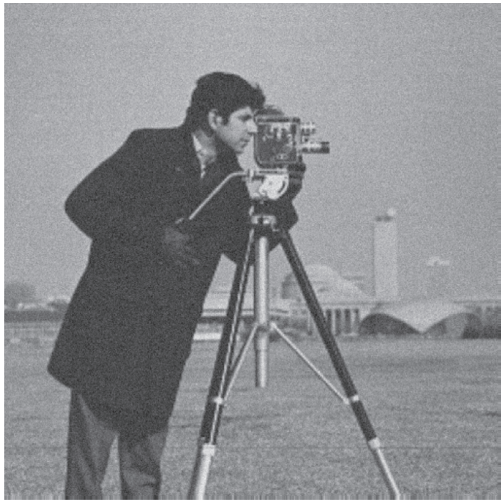

(a)

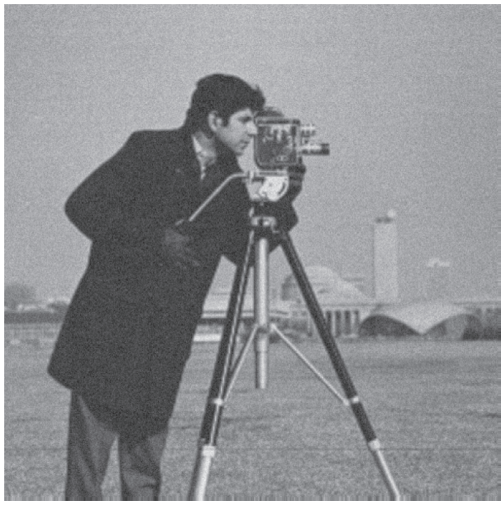

(d)

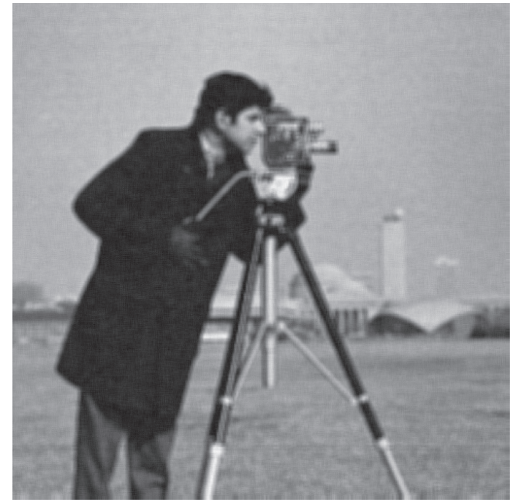

(b)

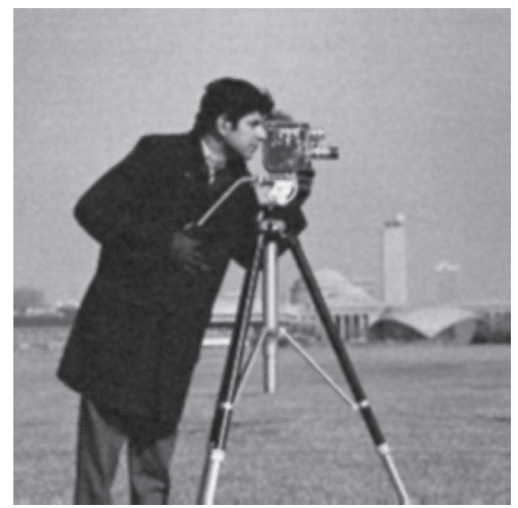

(e)

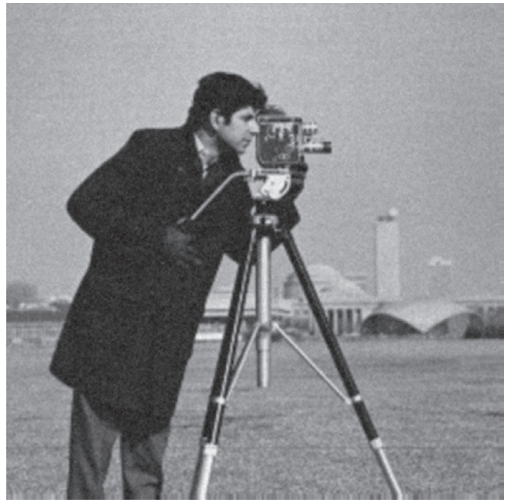

(c)

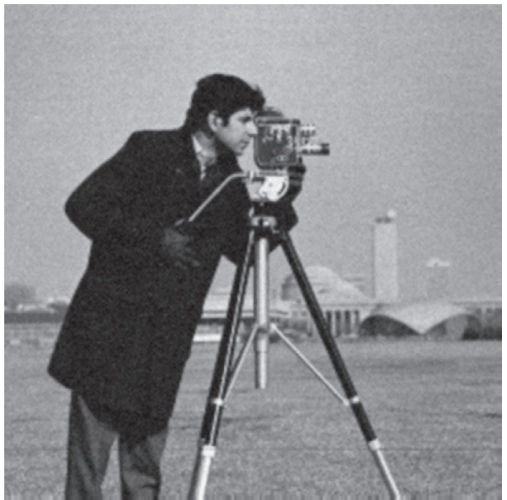

(f)

Figure 14: Comparison of different operators on "Cameraman" under Gaussian noise with variance $\sigma=15$. 


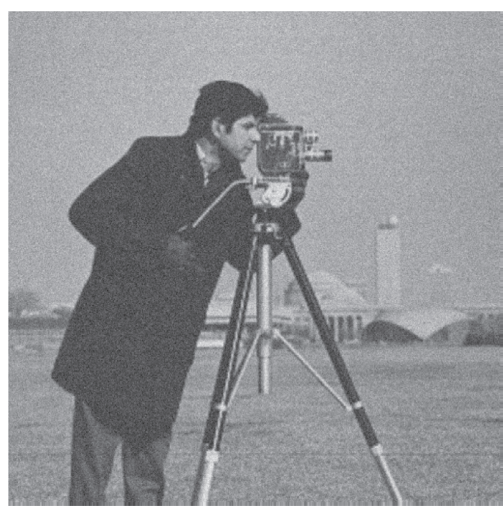

(a)

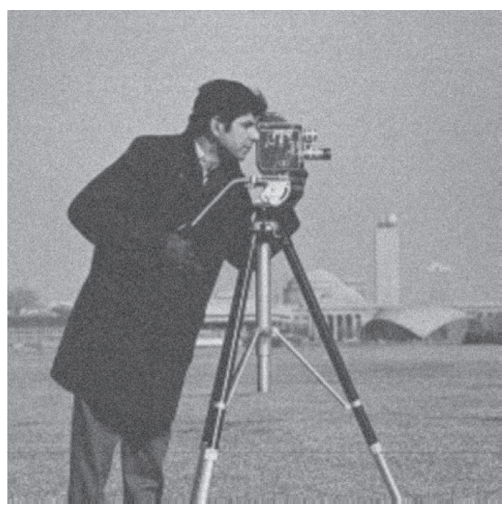

(d)

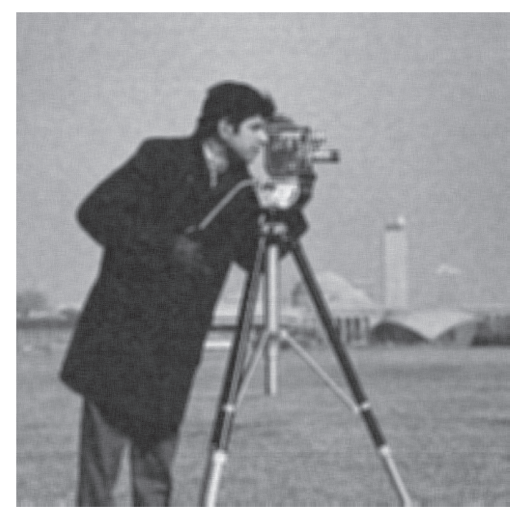

(b)

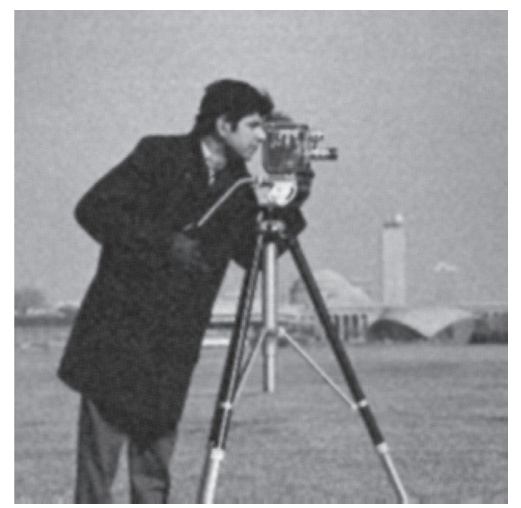

(e)

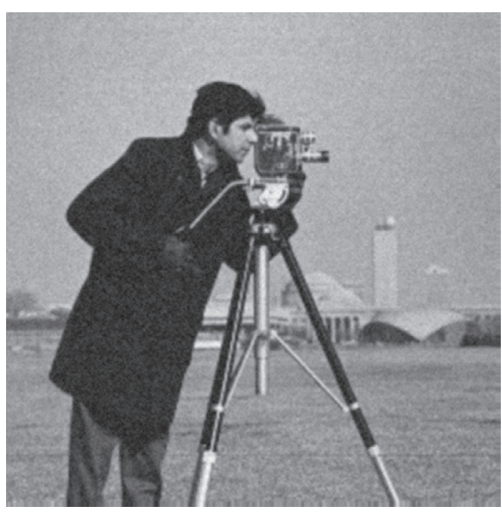

(c)

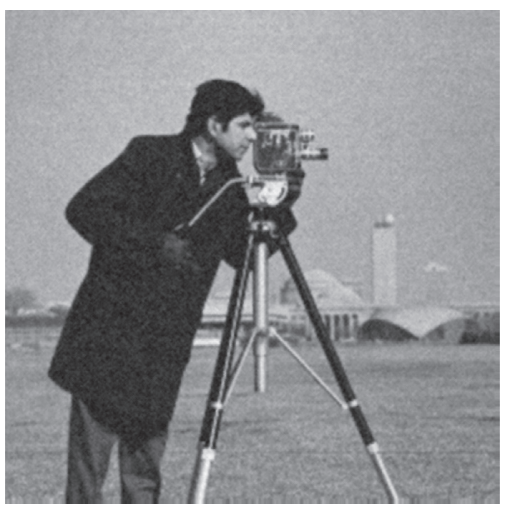

(f)

Figure 15: Comparison of different operators on "Cameraman" under Gaussian noise with variance $\sigma=20$.

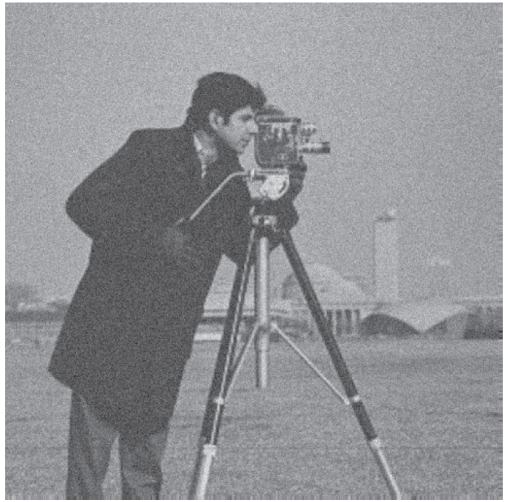

(a)

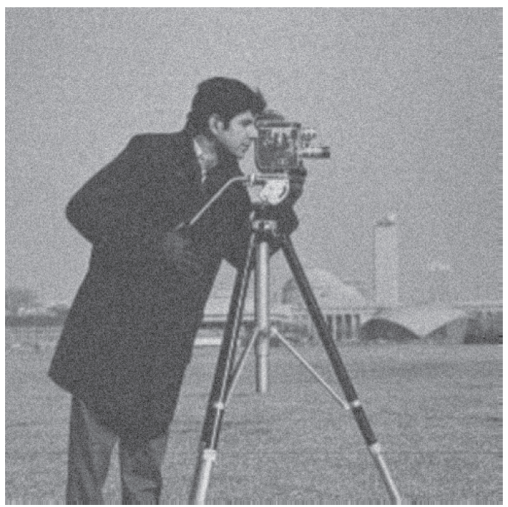

(d)

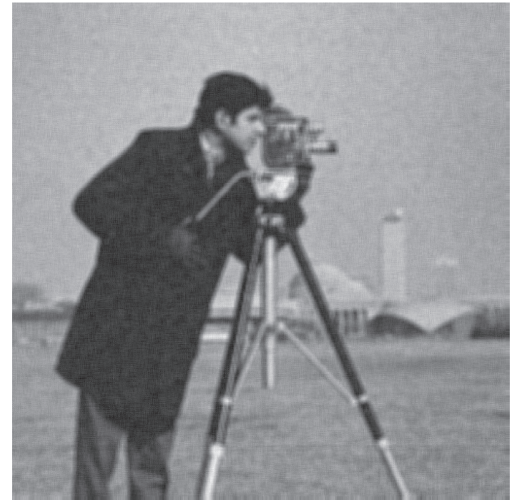

(b)

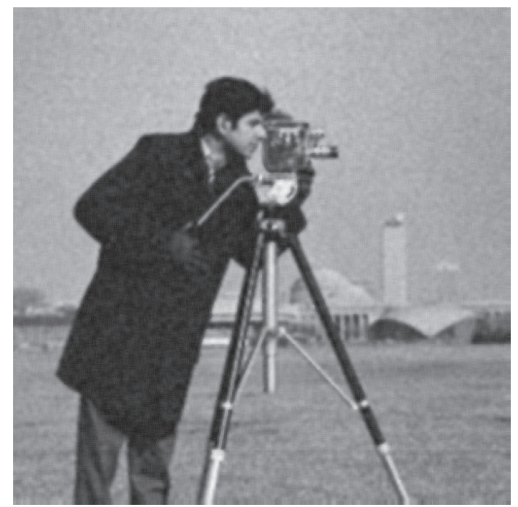

(e)

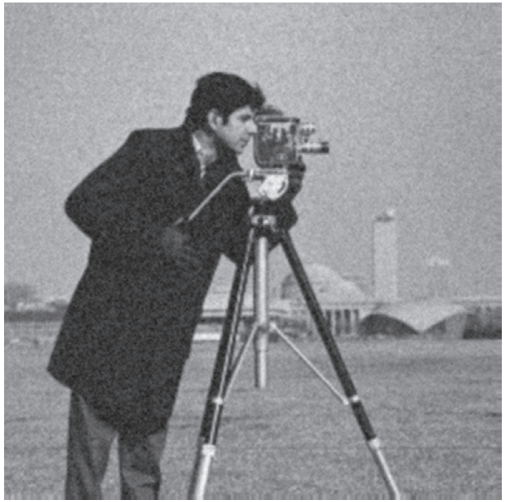

(c)

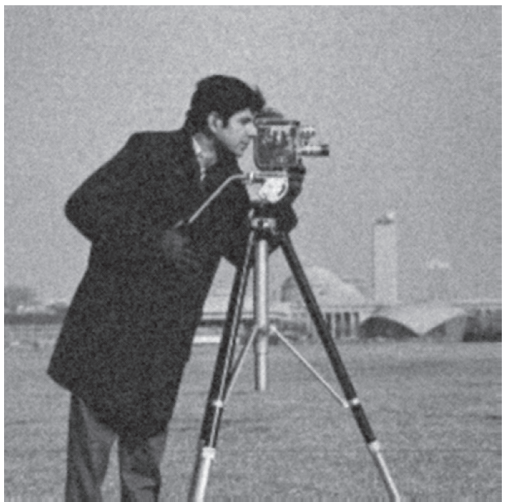

(f)

FIGURE 16: Comparison of different operators on "Cameraman" under Gaussian noise with variance $\sigma=25$. (a) Noisy image, (b) TA_ABC, (c) GL_ABC, (d) Method in [38], (e) Ada_TA_ABC, (f) Ada_GL_ABC. 
TABLE 2: Comparison of the effectiveness of different fractional operators for the Lena image.

\begin{tabular}{cccccccc}
\hline & & Noisy image & Method in [36] & TA_ABC & Ada_TA_ABC & GL_ABC & Ada_GL_ABC \\
\hline \multirow{2}{*}{$\sigma=15$} & PSNR & 24.6047 & 25.8887 & 26.6857 & 28.3095 & 30.6827 & 31.2764 \\
& SSIM & 0.4466 & 0.498 & 0.6325 & 0.7147 & 0.7498 & 0.8126 \\
\hline \multirow{2}{*}{$\sigma=20$} & PSNR & 22.1006 & 23.6471 & 26.0032 & 27.6077 & 29.2995 & 29.9716 \\
& SSIM & 0.3392 & 0.3953 & 0.556 & 0.6524 & 0.6924 & 0.7482 \\
\hline \multirow{2}{*}{$\sigma=25$} & PSNR & 20.1839 & 22.0718 & 25.3131 & 27.0041 & 28.3211 & 28.7544 \\
& SSIM & 0.2682 & 0.3296 & 0.4895 & 0.5979 & 0.6516 & 0.6845 \\
\hline
\end{tabular}

TABLE 3: Comparison of the effectiveness of different fractional operators for the Elaine image.

\begin{tabular}{cccccccc}
\hline & & Noisy image & Method in [38] & TA_ABC & Ada_TA & GL_ABC & Ada_GL_ABC \\
\hline \multirow{2}{*}{$\sigma=15$} & PSNR & 24.602 & 25.8 & 29.1284 & 30.5185 & 31.5693 & 32.5138 \\
& SSIM & 0.4239 & 0.4754 & 0.6484 & 0.7174 & 0.7575 & 0.8033 \\
\hline \multirow{2}{*}{$\sigma=20$} & PSNR & 22.1297 & 23.4999 & 28.0504 & 29.5162 & 30.4062 & 30.8692 \\
& SSIM & 0.3101 & 0.363 & 0.574 & 0.66 & 0.714 & 0.739 \\
\hline \multirow{2}{*}{$\sigma=25$} & PSNR & 20.1507 & 21.7676 & 26.9874 & 28.542 & 28.8114 & 29.3865 \\
& SSIM & 0.233 & 0.2867 & 0.5048 & 0.6024 & 0.6385 & 0.6719 \\
\hline
\end{tabular}

TABle 4: Comparison of the effectiveness of different fractional operators for the Goldhill image.

\begin{tabular}{clcccccc}
\hline & & Noisy image & Method in [38] & TA_ABC & Ada_TA_ABC & GL_ABC & Ada_GL_ABC \\
\hline \multirow{2}{*}{$\sigma=15$} & PSNR & 24.6053 & 25.5984 & 25.8959 & 26.7892 & 29.265 & 29.8402 \\
& SSIM & 0.5246 & 0.562 & 0.5339 & 0.6021 & 0.7312 & 0.7831 \\
\hline \multirow{2}{*}{$\sigma=20$} & PSNR & 22.0996 & 23.2623 & 25.2557 & 26.3092 & 28.5353 & 28.9227 \\
& SSIM & 0.4058 & 0.449 & 0.4725 & 0.5579 & 0.7035 & 0.7343 \\
\hline \multirow{2}{*}{$\sigma=25$} & PSNR & 20.1693 & 21.6197 & 24.6607 & 25.8326 & 27.6622 & 27.9387 \\
& SSIM & 0.3205 & 0.3707 & 0.4226 & 0.5176 & 0.6634 & 0.6823 \\
\hline
\end{tabular}

TABLE 5: Comparison of the effectiveness of different fractional operators for the Pepper image.

\begin{tabular}{cccccccc}
\hline & & Noisy image & Method in [38] & TA_ABC & Ada_TA_ABC & GL_ABC & Ada_GL_ABC \\
\hline \multirow{2}{*}{$\sigma=15$} & PSNR & 24.6089 & 25.5672 & 26.6575 & 27.508 & 29.9746 & $\mathbf{3 0 . 3 1 4 4}$ \\
& SSIM & 0.4525 & 0.4913 & 0.6472 & 0.7039 & 0.7407 & $\mathbf{0 . 7 9 7 9}$ \\
\hline \multirow{2}{*}{$\sigma=20$} & PSNR & 22.1164 & 23.3792 & 26.0054 & 27.0188 & 28.8524 & $\mathbf{2 9 . 3 2 3 8}$ \\
& SSIM & 0.3451 & 0.3904 & 0.576 & 0.6504 & 0.6927 & $\mathbf{0 . 7 3 8 3}$ \\
\hline \multirow{2}{*}{$\sigma=25$} & PSNR & 20.1598 & 21.6034 & 25.2919 & 26.4364 & 27.72 & $\mathbf{2 8 . 2 1 2 1}$ \\
& SSIM & 0.2723 & 0.3178 & 0.5095 & 0.5944 & 0.6326 & $\mathbf{0 . 6 7 3 3}$ \\
\hline
\end{tabular}

TABle 6: Comparison of the effectiveness of different fractional operators for the Cameraman image.

\begin{tabular}{cccccccc}
\hline & & Noisy image & Method in [38] & TA_ABC & Ada_TA_ABC & GL_ABC & Ada_GL_ABC \\
\hline \multirow{2}{*}{$\sigma=15$} & PSNR & 24.6171 & 25.7532 & 25.724 & 28.3601 & 30.9423 & 31.5346 \\
& SSIM & 0.4084 & 0.4524 & 0.6394 & 0.7668 & 0.7398 & 0.8214 \\
\hline \multirow{2}{*}{$\sigma=20$} & PSNR & 22.1178 & 23.6034 & 25.1439 & 27.7603 & 29.5537 & 30.0694 \\
& SSIM & 0.3116 & 0.3615 & 0.5502 & 0.7002 & 0.6902 & 0.7429 \\
\hline \multirow{2}{*}{$\sigma=25$} & PSNR & 20.1543 & 21.7478 & 24.5397 & 26.8904 & 28.3857 & 28.7435 \\
& SSIM & 0.2465 & 0.2923 & 0.4724 & 0.6241 & 0.635 & 0.6674 \\
\hline
\end{tabular}

TABLE 7: Comparison of the entropy of different fractional operators for all images.

\begin{tabular}{|c|c|c|c|c|c|c|c|c|c|c|c|c|c|c|c|}
\hline \multirow[b]{2}{*}{$\Sigma$} & \multicolumn{3}{|c|}{ Lena } & \multicolumn{3}{|c|}{ Elaine } & \multicolumn{3}{|c|}{ Goldhill } & \multicolumn{3}{|c|}{ Peppers } & \multicolumn{3}{|c|}{ Cameraman } \\
\hline & 15 & 20 & 25 & 15 & 20 & 25 & 15 & 20 & 25 & 15 & 20 & 25 & 15 & 20 & 25 \\
\hline $\begin{array}{l}\text { Original } \\
\text { image }\end{array}$ & & 7.4456 & & & 7.5001 & & & 7.4778 & & & 7.5715 & & & 7.0480 & \\
\hline $\begin{array}{l}\text { Method in } \\
{[36]}\end{array}$ & 7.6008 & 7.6512 & 7.6891 & 7.5873 & 7.6181 & 6525 & 7.6079 & 7.6478 & 7.6744 & 7.6767 & 7.6973 & 7.7121 & 7.3948 & 7.4416 & 7.48 \\
\hline TA_ABC & 7.44 & 7.4675 & 7.4989 & 7.4760 & 7.4874 & 7.5084 & 7.4494 & 7.4734 & 7.4956 & 7.6141 & 7.6268 & 7.6412 & 7.2339 & 7.2925 & 7.334 \\
\hline $\begin{array}{l}\text { Ada_TA_T } \\
\text { ABC }\end{array}$ & 7.4298 & 7.4515 & 7.4788 & 7.4734 & 7.4808 & 7.4979 & 7.4427 & 7.4621 & 7.4800 & 7.6071 & 7.6183 & 7.6302 & 7.1802 & 7.2381 & 7.288 \\
\hline GL_ABC & & 7.5028 & 7.5 & . & 7.51 & & 7.5 & 7.51 & & 7.62 & 7.6329 & & 7.2693 & 7.2965 & 7.322 \\
\hline Ada_GL_ABC & 7.4508 & 7.4744 & 7.5038 & 7.4944 & 7.5042 & 7.5205 & 7.7405 & 7.4901 & 7.5101 & 7.6101 & 7.6224 & 7.6365 & 7.2111 & 7.2674 & 7.3085 \\
\hline
\end{tabular}


the entropy of test images, as shown in Table 7. The entropy of images processed by the Ada_GL_ABC mask is closer to that of the original images than that got by other masks, but it is also higher than that of the original images. The results demonstrate that the detailed information of images is preserved while denoising. For Figures 2-16, (e) and (f) are clearer than the others by visual evaluation. TA_ABC and GL_ABC have been improved by the proposed adaptive function. According to the quantitative indicators shown in the tables, the Ada_GL_ABC mask has better denoising and detail-preserving ability than other masks. Furthermore, Ada_GL_ABC and Ada_TA_ABC masks are robust for different intensity noise by the analysis of the results. The effectiveness of our proposed adaptive operator can be proved from the two aspects of vision and evaluation index.

\section{Conclusions}

In this paper, the adaptive denoising mask is proposed based on Atangana-Baleanu derivatives. The key to this method is the calculation of order. The order is determined by the intensity of the gradient, global entropy, local entropy, and local variance. These variables represent the whole and local information of the image. To protect the texture details, we design the adaptive order integral operator considering global and local information. This operator can produce smaller orders in the image edge and texture details while larger orders in the smooth region. The proposed function is used to improve the GL_ABC mask and TA_ABC mask operator. We test the effectiveness of our proposed algorithm on multiple images. From a visual point of view, the denoising ability of Ada_TA_ABC and Ada_GL_ABC are reliable. Compared with other operators by the evaluation indicators, the Ada_GL_ABC operator works better. And, the PSNR and the SSIM are all higher under different intensities of noise. The information entropy index of the image processed by Ada_TA_ABC and Ada_GL_ABC operators is closer to the original images. The entropy of image filtered by Ada_GL_ABC mask is slightly larger, which indicates that Ada_GL_ABC mask can preserve texture details. These experimental results confirm that GL_ABC and TA_ABC have all been improved. And, the proposed adaptive function has a certain degree of universality.

\section{Data Availability}

The test images used to support the findings of this study are included within the article.

\section{Conflicts of Interest}

The authors declare no conflicts of interest.

\section{Acknowledgments}

This work was supported by the Science and Technology Research Project of Higher Education of Hebei Province (grant nos. QN2019069 and QN2018116), the Research Foundation of Hebei University of Economics and Business (grant nos. 2018QZ05, 2018QZ04, 2019YB11, 2019YB08, and 2020YB17).

\section{References}

[1] S. Zhou, X. Lin, H. Li et al., "Chaotic synchronization of a fractional-order system based on washout filter control," Communications in Nonlinear Science and Numerical Simulation, vol. 16, no. 3, pp. 1533-1540, 2011.

[2] M. A. Khan and A. Atangana, "Modeling the dynamics of novel coronavirus (2019-nCov) with fractional derivative," Alexandria Engineering Journal, vol. 59, no. 4, pp. 2379-2389, 2020.

[3] G. Wang, Y. Liu, Y. Wu et al., "Symmetry analysis for a seventh-order generalized $\mathrm{KdV}$ equation and its fractional version in fluid mechanics," Fractals, vol. 28, no. 3, pp. 127-134, 2020.

[4] T. J. Freeborn, "A survey of fractional-order circuit models for biology and biomedicine," IEEE Journal on Emerging and Selected Topics in Circuits and Systems, vol. 3, no. 3, pp. 416-424, 2013.

[5] B. N. Lundstrom, M. H. Higgs, W. J. Spain, and A. L. Fairhall, "Fractional differentiation by neocortical pyramidal neurons," Nature Neuroscience, vol. 11, no. 11, pp. 1335-1342, 2008.

[6] Z. Wang, X. Huang, and G. Shi, "Analysis of nonlinear dynamics and chaos in a fractional order financial system with time delay," Computers \& Mathematics with Applications, vol. 62, no. 3, pp. 1531-1539, 2011.

[7] X. Hu, Q. Song, M. Ge, and R. Li, "Fractional-order adaptive fault-tolerant control for a class of general nonlinear systems," Nonlinear Dynamics, vol. 101, no. 1, pp. 379-392, 2020.

[8] H. A. Jalab, R. W. Ibrahim, A. Ahmed et al., "Image denoising algorithm based on the convolution of fractional Tsallis entropy with the Riesz fractional derivative," Neural Computing and Applications, vol. 28, no. S1, pp. 217-223, 2017.

[9] F. Hu, S. Si, H. S. Wong, B. Fu, M. Si, and H. Luo, "An adaptive approach for texture enhancement based on a fractional differential operator with non-integer step and order," Neurocomputing, vol. 158, no. 22, pp. 295-306, 2015.

[10] J. Bai and X.-C. Feng, "Fractional-order anisotropic diffusion for image denoising," IEEE Transactions on Image Processing, vol. 16, no. 10, pp. 2492-2502, 2007.

[11] Y. Pu, P. Siarry, A. Chatterjee et al., "A fractional-order variational framework for retinex: fractional-order partial differential equation-based formulation for multi-scale nonlocal contrast enhancement with texture preserving," IEEE Transactions on Image Processing, vol. 27, pp. 1214-1229, 2017.

[12] Y. Pu, "Application of fractional differential approach to digital image processing," Advanced Engineering Sciences, vol. 39, no. 3, pp. 124-132, 2007.

[13] Y. Pu, J. Zhou, and X. Yuan, "Fractional differential mask: a fractional differential-based approach for multiscale texture enhancement," IEEE Transactions on Image Processing, vol. 19, no. 2, pp. 491-511, 2010.

[14] J. Guan, J. Ou, Z. Lai et al., "The medical image enhancement method based on the fractional order derivative and the directional derivative," International Journal of Pattern Recognition and Artificial Intelligence, vol. 32, Article ID 1857001, 2018.

[15] C. Wang, L. Lan, and S. Zhou, "Grunwald-Letnikov based adaptive fractional differential algorithm on image texture enhancing," Journal of Computational Information Systems, vol. 9, no. 2, pp. 445-454, 2013.

[16] A. Azarang and H. Ghassemian, "Application of fractionalorder differentiation in multispectral image fusion," Remote Sensing Letters, vol. 9, no. 1, pp. 91-100, 2017. 
[17] A. Saadia and A. Rashdi, "Incorporating fractional calculus in echo-cardiographic image denoising," Computers \& Electrical Engineering, vol. 67, pp. 134-144, 2018.

[18] W. Gao, P. Veeresha, D. G. Prakasha et al., "New numerical results for the time-fractional phi-four equation using a novel analytical approach," Symmetry, vol. 12, Article ID 478, 2020.

[19] H. J. Haubold, A. M. Mathai, and R. K. Saxena, "New numerical simulation for fractional Benney-Lin equation arising in falling film problems using two novel techniques," $\mathrm{Nu}$ merical Methods for Partial Differential Equations, vol. 37, no. 1, pp. 210-243, 2021.

[20] K. M. Owolabi and Z. Hammouch, "Spatiotemporal patterns in the Belousov-Zhabotinskii reaction systems with Atangana-Baleanu fractional order derivative," Physica A: Statistical Mechanics and Its Applications, vol. 523, pp. 1072-1090, 2019.

[21] K. M. Owolabi and Z. Hammouch, "Mathematical modeling and analysis of two-variable system with noninteger-order derivative," Chaos, vol. 29, no. 1, Article ID 013145, 2019.

[22] J. D. Djida, A. Atangana, and I. Area, "Numerical computation of a fractional derivative with non-local and non-singular kernel," Mathematical Modelling of Natural Phenomena, vol. 12, no. 3, pp. 4-13, 2017.

[23] D. Baleanu and A. Fernandez, "On some new properties of fractional derivatives with Mittag-Leffler kernel," Communications in Nonlinear Science and Numerical Simulation, vol. 59, pp. 444-462, 2017.

[24] H. J. Haubold, A. M. Mathai, and R. K. Saxena, "Mittag-leffler functions and their applications," Journal of Applied Mathematics, vol. 2011, Article ID 298628, 51 pages, 2011.

[25] J. E. Solís-Pérez, J. F. Gómez-Aguilar, R. F. Escobar-Jiménez, and J. Reyes-Reyes, "Blood vessel detection based on fractional Hessian matrix with non-singular Mittag-Leffler Gaussian kernel," Biomedical Signal Processing and Control, vol. 54, Article ID 101584, 2019.

[26] B. Ghanbari and A. Atangana, "A new application of fractional Atangana-Baleanu derivatives: designing ABC-fractional masks in image processing," Physica A Statistical Mechanics \& Its Applications, vol. 542, Article ID 123516, 2019.

[27] G. Huang, L. Xu, and Y. Pu, "Summary of research on image processing using fractional calculus," Application Research of Computers, vol. 29, no. 2, pp. 414-426, 2012.

[28] A. Atangana and D. Baleanu, "New fractional derivatives with nonlocal and non-singular kernel: theory and application to heat transfer model," Thermal Science, vol. 20, no. 2, pp. 763-769, 2016.

[29] K. B. Oldham and J. Spanier, "The fractional calculus," Mathematical Gazette, vol. 56, no. 247, pp. 396-400, 1974.

[30] J. Yu, L. Tan, S. Zhou et al., "Image denoising algorithm based on entropy and adaptive fractional order calculus operator," IEEE Access, vol. 5, pp. 12275-12285, 2017.

[31] J. Prehl, F. Boldt, K. H. Hoffmann et al., "Symmetric fractional diffusion and entropy production," Entropy, vol. 18, no. 8, p. 275, 2016.

[32] B. Qiao, L. Jin, and Y. Yang, “An adaptive algorithm for grey image edge detection based on grey correlation analysis," in Proceedings of the International Conference on Computational Intelligence \& Security, pp. 470-474, Wuxi, China, December 2017.

[33] W. Xie, M. You, and Y. Zhou, "Guided image filter and application based on adaptive fractional-order differential," Application Research of Computers, vol. 34, no. 001, pp. 283-286, 2017.
[34] N. He, J.-B. Wang, L.-L. Zhang, and K. Lu, "An improved fractional-order differentiation model for image denoising," Signal Processing, vol. 112, pp. 180-188, 2015.

[35] W. Zhou, A. C. Bovik, H. R. Sheikn et al., "Image quality assessment: from error visibility to structural similarity," IEEE Transactions on Image Processing, vol. 13, no. 4, pp. 600-612, 2004.

[36] B. Li and W. Xie, "Image enhancement and denoising algotithms based on adaptive fractional differential and integral," Systems Engineering and Electronics, vol. 38, pp. 185-192, 2016. 